\title{
Probabilistic Voting in the Spatial Model of Elections: The Theory of Office-motivated Candidates
}

\author{
Jeffrey S. Banks
}

Division of Humanities and Social Sciences

California Institute of Technology

John Duggan

Department of Political Science

and Department of Economics

University of Rochester

July 4, 2003 


\begin{abstract}
We unify and extend much of the literature on probabilistic voting in two-candidate elections. We give existence results for mixed and pure strategy equilibria of the electoral game. We prove general results on optimality of pure strategy equilibria vis-a-vis a weighted utilitarian social welfare function, and we derive the well-known "mean voter" result as a special case. We establish broad conditions under which pure strategy equilibria exhibit "policy coincidence," in the sense that candidates pick identical platforms. We establish the robustness of equilibria with respect to variations in demographic and informational parameters. We show that mixed and pure strategy equilibria of the game must be close to being in the majority rule core when the core is close to non-empty and voters are close to deterministic. This contraverts the notion that the median (in a one-dimensional model) is a mere "artifact." Using an equivalence between a class of models including the binary Luce model and a class including additive utility shock models, we then derive a general result on optimality vis-a-vis the Nash social welfare function.
\end{abstract}




\section{Introduction}

The spatial model of elections, originating in the work of Hotelling (1929), Downs (1957), and Black (1958), is perhaps the simplest formalization of democratic politics: each of two candidates adopt policy platforms before an election, voters cast their votes, and the winner implements his or her platform. Theorists differ, of course, in their treatments of the details of the model. Common to most work on the spatial model is the assumption that candidates can credibly commit to their campaign promises. ${ }^{1}$ Beyond that, more candidates may be permitted; the policy space may be finite, one-dimensional, or even multi-dimensional; the set of voters may be finite or a continuum, with preferences satisfying assorted conditions; the candidates' objectives may be to maximize their probabilities of winning, or they may seek to maximize their pluralities, or they may be motivated by policy preferences, or their objectives may be some mix of these alternatives. In addition, the nature of the voting decision is at issue: in much work on the spatial model, the strategy of each voter is modelled as the solution of a known maximization problem depending on the platforms of the candidates, allowing the candidates to anticipate with certainty the voters' responses to different policy positions.

Because political candidates are unlikely in reality to be able to predict election returns with certainty, let alone the vote of any one voter, and because models of that sort often suffer from difficult technical problems, a theoretical literature has treated voting as a random variable, i.e., it is assumed that voters vote probabilistically from the perspective of candidates. This approach is consistent with the rational choice approach to voting and elections, if we regard this randomness as arising from unobserved parameters in the maximization problems of voters. As we discuss in Section 2, much of the theory developed in this paper is motivated by one such model in which randomness enters the decision of a voter through a shock, or "bias" term, added to the voter's payoffs from the candidates' policy platforms. Specifically, we might suppose that each candidate possesses non-policy characteristics, or there are some policy dimensions on which the candidates cannot commit, that are relevant to the voter. The voter's utility from having a candidate take office is then equal to the sum of the utility from the candidate's policy platform and a utility increment, unobserved by the candidates, due to these fixed factors. The voter simply votes for the candidate offering the highest utility.

Though the literature on probabilistic voting has a long tradition in political science and political economy, the many models considered in that literature lack a unifying framework. As a consequence, the results of the models are difficult to compare, and superficial similarities and differences are difficult to interpret. Our objective in this paper is to supply such a framework, while generalizing many known results and providing a new, rigorous analysis of continuity of equilibria with respect to changes in the electoral environment. Our analysis is limited in two respects: we allow for only two candidates, and we assume that candidates maximize expected plurality. The first limitation is a widely considered benchmark. The second is common in the probabilistic voting literature, and is sometimes consistent with probability of winning maximization, ${ }^{2}$ but we limit ourselves to office-motivated candidates.

\footnotetext{
${ }^{1} \mathrm{~A}$ branch of the literature considers citizen-candidates, who cannot commit to their platforms. See Osborne and Slivinski (1996), Besley and Coate (1997), and Banks and Duggan (2002).

${ }^{2}$ See Patty $(2002,2003)$ and Duggan $(2000)$ and references found there for results on the equivalence of
} 
Within the confines of those two qualifications, we cast our net quite widely: we allow for a multi-dimensional policy space, a finite set or continuum of voter types, and general concave voter utility functions.

Our analysis ranges over several traditional topics of interest: existence and uniqueness of electoral equilibria, the characterization of equilibrium outcomes as social welfare maxima, and the incentives for candidates to adopt identical platforms in equilibrium, a phenonomenon we refer to as "policy coincidence." In addition, we provide several results on the robustness of equilibrium platforms with respect to electoral parameters, such as the distribution of voter types. And we consider the nature of equilibrium platforms when the set of unbeatable policy positions, i.e., the core ${ }^{3}$ is close to non-empty and voting behavior is close to deterministic. Throughout, we have attempted to disentangle these issues as much as possible. For example, some authors prove existence and uniqueness of pure strategy equilibria in the models they consider, simultaneously showing that the candidates choose the same platform in equilibrium, and also characterizing that platform as a social welfare optimum. In contrast, we first give general conditions under which mixed strategy equilibria exist, then stronger conditions under which pure strategy equilibria exist, and then stronger conditions guaranteeing uniqueness. Our results on existence and some on uniqueness of pure strategy equilibria involve continuity and concavity conditions on the voters' probability of voting functions and hark back to the work of Hinich, Ledyard, and Ordeshook $(1972,1973)$.

We take up the issue of optimality of equilibria and find weak conditions under which equilibria correspond to utilitarian social welfare optima: in the Additive Bias Model, for example, if the voter' biases are identically and continuously distributed, and if voters have concave utility functions, then every pure strategy equilibrium exhibiting policy coincidence maximizes the sum of the voters' utility functions. Our general theorems unify results by Hinich (1978), Lindbeck and Weibull $(1987,1993)$, and Coughlin (1992). We then take up the issue of policy coincidence, found in numerous theoretical studies of elections, including Hotelling (1929). Combined with our existence results, a simple symmetry condition on probability of voting functions yields at least one equilibrium exhibiting policy coincidence. But the phenomenon of policy coincidence is much deeper than this. We drop the assumption of candidate symmetry and find that pure strategy equilibria necessarily exhibit policy coincidence under very weak assumptions: in the Additive Bias Model, for example, if voters have strictly concave utility functions, then every pure strategy equilibrium exhibits policy coincidence.

We offer a general result on the robustness of equilibrium platforms with respect to variations in underlying electoral parameters, an issue broached by Calvert (1985). ${ }^{4}$ The problem is formulated generally enough that we can consider variations in the distribution of voter types, or we can consider variations in the voters' probability of vote functions. We show, for example, that if voter types are distributed normally with some variance, say $\sigma$, then a small change in $\sigma$ cannot lead to equilibrium outcomes far away from the equilibria of the original model - a desirable property when the parameters of a model are difficult

\footnotetext{
expected plurality and probability of winning maximization.

${ }^{3}$ Policy positions in the core are sometimes referred to as "Condorcet winners."

${ }^{4}$ Technically, we prove that the equilibrium correspondence of the game between the candidates has closed graph.
} 
to observe in applications.

In the model with deterministic voters, the core, if non-empty, consists of exactly the platforms used in pure or mixed strategy equilibria. But emptiness of the core is typical when the space of policy positions is multi-dimensional, and this equivalence only obtains in the deterministic voting case. Thus, by itself, this characterization is of limited use in understanding the location of equilibrium platforms in multi-dimensional models with probabilistic voters. We show, however, that if voter preferences are such that the core is "close" (in a precise topological sense) to being non-empty and voters are "close" to being deterministic, then pure and mixed strategy equilibrium platforms must be "close" to being in the core. These core convergence results shed light on an old debate, initiated by Hinich $(1977,1978)$ and taken up by Kramer $(1978)$, about the fragility of the median in the onedimensional model with deterministic voters: an implication of our results is that, when a small amount of randomness is added to the choices of voters, the median will typically no longer constitute a pure strategy equilibrium, and (pure or mixed strategy) equilibrium platforms must be close to the median. The latter conclusion contradicts Hinich's (1978) Theorem 2, which claims that equilibria would be at the mean (not the median), and corroborates Kramer's claim of median robustness.

In much of the paper, we assume that voters respond to differences in utility levels, and accordingly we characterize equilibrium platforms as utilitarian social welfare optima. Finally, we consider a class of models in which voters respond to ratios of utility levels, including, for example, the Binary Luce Model used by Coughlin and Nitzan (1981) and Coughlin (1992). Exploiting a tight connection between the two classes of models, we show how results for Utility Difference Models can be translated directly into results for Utility Ratio Models. For example, we derive the results of the latter authors on Nash social welfare optima from our results on utilitarian social welfare optima. Moreover, our result holds for the Additive Bias Model if the voter' biases are identically and continuously distributed and utility functions are log-concave - the assumption of the Luce functional form for probability of vote functions is unneeded.

Two notable papers on probabilistic voting that are not covered by our analysis are Ledyard (1984) and, recently, McKelvey and Patty (1999). In both papers, the probability a voter votes for one candidate or another, fixing their policy platforms, is determined in the equilibrium of a game of incomplete information among the voters. In Ledyard's paper, each voter has a private cost of voting which, along with the probability of being pivotal given others' strategies, determines the voter's decision to vote or abstain. This generates probability of vote functions for the candidates and defines the electoral game. Ledyard shows that there is a unique electoral equilibrium, and that the policy platforms adopted in equilibrium maximize the sum of voters' utilities. Though these themes appear later in our paper, Ledyard's framework is quite different from ours: he assumes candidates maximize probability of winning, rather than expected plurality, and his probability of vote functions would be likely to fail many of our conditions. Thus, his results cannot be obtained from ours.

McKelvey and Patty (2003), unlike other work cited above, allow for multiple candidates. They suppose that each voter has a private value attached to voting for the different candidates, above and beyond the utility from their policy platforms, and they show that, 
for a large enough electorate, there exists a pure strategy equilibrium in which all candidates locate at the utilitarian social welfare optimum. In the two-candidate case with no abstention, their model appears to be quite similar to the Additive Bias Model, as the value of voting for a candidate looks like an additive utility shock. In the model of McKelvey and Patty, however, the utility increment for a candidate is realized if the voter merely votes for the candidate, regardless of whether the candidate wins: a voter must consider the sum of the utility from a candidate's policy platform, weighted by the probability of being pivotal, and the value of voting for that candidate. Because pivot probabilities are determined in equilibrium, their probability of vote functions will not typically satisfy our conditions, and their results cannot be obtained from ours.

\section{The Framework}

\subsection{The Electoral Game}

Two candidates (or parties) $A$ and $B$ simultaneously choose points $x_{A}$ and $x_{B}$ in a multidimensional policy space $X \subseteq \Re^{n}$. A measure space of voters then decide between the candidates in a majority rule election. We suppose the electorate is partitioned into a probability space $(T, \mathcal{T}, \mu)$ of types: the probability a voter's type lies in (measurable) $S \subseteq T$ is $\mu(S)$. We take as given a family of functions $P_{t}^{C}: X \times X \rightarrow \Re, C \in\{A, B\}$, and where we impose the following minimal mathematical structure:

- $P_{t}^{A}\left(x_{A}, x_{B}\right)$ is jointly measurable in $t$ and $x_{A}$ for each fixed $x_{B}$.

- $P_{t}^{B}\left(x_{A}, x_{B}\right)$ is jointly measurable in $t$ and $x_{B}$ for each fixed $x_{A}$.

- $-1 \leq P_{t}^{C}\left(x_{A}, x_{B}\right) \leq 1$ for each $\left(t, x_{A}, x_{B}\right)$ and each $C$.

These functions are meant to capture uncertainty underlying the decisions of voters. It may be that, in contrast to the usual rational choice assumption, voters actually behave randomly; or the candidates may have subjective beliefs about how voters vote; or, the explanation we prefer, the candidates may face objective probabilities derived from an underlying environment of incomplete information. We give specific examples in the next subsection.

At this point, we have not given the functions $P_{t}^{C}$ a specific interpretation. It could be, for example, that $P_{t}^{C}\left(x_{A}, x_{B}\right)$ is the probability that a type $t$ voter would vote for $C$ given platforms $\left(x_{A}, x_{B}\right)$; or it could be the expected share of votes for $C$ from type $t$ voters; or it could be the expected plurality share for $C$ from type $t$ voters, where the share is calculated as a proportion of the total number of type $t$ voters. We will select the latter interpretation by maintaining the following assumption.

- $P_{t}^{A}\left(x_{A}, x_{B}\right)+P_{t}^{B}\left(x_{A}, x_{B}\right)=0$ for each $\left(t, x_{A}, x_{B}\right)$.

We then define the electoral game by identifying each candidate's payoff with the candidate's expected plurality share in the election. Thus, $\left(x_{A}, x_{B}\right)$ is a pure strategy electoral 
equilibrium if $x_{A}$ solves

$$
\max _{x \in X} \int_{T} P_{t}^{A}\left(x, x_{B}\right) \mu(d t)
$$

and $x_{B}$ solves

$$
\max _{x \in X} \int_{T} P_{t}^{B}\left(x_{A}, x\right) \mu(d t) .
$$

We say a platform pair $\left(x_{A}, x_{B}\right)$ exhibits policy coincidence if $x_{A}=x_{B}$. We consider alternative objective functions that have also been used to model office-motivation at the end of this section.

We will also consider equilibria in which each candidate $C$ may use a mixed strategy, a Borel probably measure, denoted $\pi_{C}$, on the policy space $X$. Formally, letting $\Pi$ denote the space of Borel probability measures on $X$, a pair $\left(\pi_{A}, \pi_{B}\right)$ is a mixed strategy electoral equilibrium if $\pi_{A}$ solves

$$
\max _{\pi \in \Pi} \int_{T} \int_{X} \int_{X} P_{t}^{A}\left(x_{A}, x_{B}\right) \pi\left(d x_{A}\right) \pi_{B}\left(d x_{B}\right) \mu(d t)
$$

and $\pi_{B}$ solves

$$
\max _{\pi \in \Pi} \int_{T} \int_{X} \int_{X} P_{t}^{A}\left(x_{A}, x_{B}\right) \pi\left(d x_{B}\right) \pi_{A}\left(d x_{A}\right) \mu(d t) .
$$

Equivalently, $\pi_{C}$ must put probability one on the platforms that maximize $C$ 's expected plurality share. When considering continuity properties of mixed strategy electoral equilibria, we endow the space of mixed strategy pairs with the weak* topology. ${ }^{5}$

\subsection{Special Cases of the Model}

We would like foundations for the expected plurality share functions given above that connect candidates' payoffs to voting behavior based, at least in part, on voters' policy preferences. To this end, let the policy preferences of a type $t$ voter be given by $u_{t}: X \rightarrow \Re$, where $u_{t}(x)$ is bounded and jointly measurable. Let $\mathcal{U}_{t}=\left\{u_{t}(x) \mid x \in X\right\}$ denote the range of the type $t$ voters' utility functions. In the remainder of this section, we introduce some important special cases of the general model. We begin with one in which our results do not apply.

The Random Utility Model. In its simplest form, voters' preferences are quadratic (i.e., $u_{t}(x)=-\left\|x-x^{t}\right\|^{2}$ ), where the ideal point $x^{t}$ of the type $t$ voter is drawn from a continuous distribution function $F_{t}$ on $X$. Then $P_{t}^{C}\left(x_{A}, x_{B}\right)$ is the probability that the ideal point $x^{t}$ is closer to candidate $C$ 's platform: in the one-dimensional model, for example, we have

$$
P_{t}^{A}\left(x_{A}, x_{B}\right)= \begin{cases}F_{t}\left(\frac{x_{A}+x_{B}}{2}\right) & \text { if } x_{A}<x_{B} \\ 1-F_{t}\left(\frac{x_{A}+x_{B}}{2}\right) & \text { if } x_{B}<x_{A} \\ \frac{1}{2} & \text { if } x_{A}=x_{B} .\end{cases}
$$

It is a "folk theorem" that the unique pure strategy equilibrium is for both candidates to locate at the median of the distribution $F=\int F_{t} d \mu$. Because the candidates' objective

\footnotetext{
${ }^{5}$ That is, a sequence $\left\{\pi^{m}\right\}$ of probability measures converges to a probability measure $\pi$ if and only if, for every bounded and continuous $f: X \rightarrow \Re$, we have $\int f d \pi^{m} \rightarrow \int f d \pi$.
} 
functions are clearly discontinuous here, and because continuity of the expected plurality share functions is critical for the results of this paper, our analysis does not cover the Random Utility Model.

We will focus largely on the next model and several of its special cases.

The Fixed Utility Model. The policy preferences of voters are now fixed and expected plurality shares depend on policy positions only through utilities: there exist functions $\tilde{P}_{t}^{C}: \Re^{2} \rightarrow \Re$ such that

$$
P_{t}^{C}\left(x_{A}, x_{B}\right)=\tilde{P}_{t}^{C}\left(u_{t}\left(x_{A}\right), u_{t}\left(x_{B}\right)\right),
$$

$C \in\{A, B\}$. In this case, the expected plurality share functions are weakly monotonic if, for all $u, v, u^{\prime}, v^{\prime} \in \mathcal{U}_{t}, u^{\prime}>u$ and $v^{\prime}>v$ imply

$$
\begin{aligned}
& \tilde{P}_{t}^{A}\left(u^{\prime}, v\right) \geq \tilde{P}_{t}^{A}(u, v) \\
& \tilde{P}_{t}^{B}\left(u, v^{\prime}\right) \geq \tilde{P}_{t}^{B}(u, v) .
\end{aligned}
$$

They are strictly monotonic if the above inequalities hold strictly. Note that, because they determine expected plurality shares, voter utility functions are used for more than the purpose of representing ordinal preferences over policies.

We will often suppose the voters' utility functions are concave, and we sometimes wish to make use of strict concavity properties. Rather than assume all (or even any) voters have strictly concave utility functions, we can assume aggregate strict concavity, meaning that, for all distinct $x, y \in X$ and for all $\alpha \in(0,1)$, the set of voter types $t$ such that $u_{t}(\alpha x+(1-\alpha) y)>\alpha u_{t}(x)+(1-\alpha) u_{t}(y)$ has positive measure. This weaker assumption allows us to capture economic models in which policies include allocations of private goods and voters' preferences are strictly concave in their own consumption but constant in the consumption of others. ${ }^{6}$

The Utility Difference Model. In this special case of the Fixed Utility Model, expected plurality shares depend only on the difference in utilities from the candidates' platforms: there exist functions $\hat{P}_{t}^{C}: \Re \rightarrow \Re$ such that

$$
P_{t}^{C}\left(x_{A}, x_{B}\right)=\hat{P}_{t}^{C}\left(u_{t}\left(x_{A}\right)-u_{t}\left(x_{B}\right)\right),
$$

$C \in\{A, B\}$. In the Utility Difference Model, weak monotonicity means that $\hat{P}_{t}^{A}$ is nondecreasing and $\hat{P}_{t}^{B}$ is non-increasing for all $t$, and strict monotonicity means $\hat{P}_{t}^{A}$ is strictly increasing and $\hat{P}_{t}^{B}$ is strictly decreasing for all $t$. Note the implicit assumption that Utility Differences are meaningful: scaling $u_{t}$ by a positive constant for some types can affect the objective functions of the candidates. It may be, for example, that $u_{t}(x)-u_{t}(y)$ measures the type $t$ voter's willingness to pay for $x$ over $y$ in terms of some (unmodelled) private good.

The Additive Bias Model. In a further special case of the Utility Difference Model - and one we rely on to motivate many of our assumptions - each type $t$ voter has a random utility

\footnotetext{
${ }^{6}$ The assumption is also fulfilled if voters have concave and strictly quasi-concave utility functions, and if, for all $x, y \in X$ and all $\alpha \in(0,1)$, the set of voter types $t$ such that $u_{t}(\alpha x+(1-\alpha) y)>\max \left\{u_{t}(x), u_{t}(y)\right\}$ has positive measure. For example, types may be continuously distributed on $T=X$ and $u_{t}(x)=-\|t-x\|$.
} 
bias $\beta$ in favor of candidate $B$ given by some marginal distribution function $G_{t}$, where $G_{t}(\beta)$ is jointly measurable in $(t, \beta){ }^{7}$ Then the type $t$ voter votes for $A$ if $u_{t}\left(x_{A}\right)>u_{t}\left(x_{B}\right)+\beta$, votes for $B$ if the inequality is reversed, and "flips a fair coin" in the event of equality. In general, the candidates' expected vote shares are

$$
\begin{aligned}
\hat{V}_{t}^{A}(u) & =\frac{1}{2}\left[G_{t}(u)+\lim _{u^{\prime} \uparrow u} G_{t}\left(u^{\prime}\right)\right] \\
\hat{V}_{t}^{B}(u) & =1-\frac{1}{2}\left[G_{t}(u)+\lim _{u^{\prime} \uparrow u} G_{t}\left(u^{\prime}\right)\right],
\end{aligned}
$$

and their expected plurality share functions are given by $\hat{P}_{t}^{C}=\hat{V}_{t}^{C}-\hat{V}_{t}^{C}$. Thus, weak monotonicity is automatic. Discontinuities in $G_{t}$ will lead to discontinuities in $\hat{V}_{t}^{C}$ and $\hat{P}_{t}^{C}$. If $G_{t}$ is continuous, however, then $\hat{V}_{t}^{A}=G_{t}$ and $\hat{V}_{t}^{B}=1-G_{t}$, implying

$$
\begin{aligned}
\hat{P}_{t}^{A} & =2 G_{t}-1 \\
\hat{P}_{t}^{B} & =1-2 G_{t} .
\end{aligned}
$$

Because they are positive affine transformations of the bias distributions, the assumptions we later impose on expected plurality share functions (e.g., strict monotonicity, differentiability, or concavity) have a clear interpretation in terms of the primitives of the Additive Bias Model.

The Utility Ratio Model. In this alternative to the Utility Difference Model, it is assumed that $u_{t}(x)>0$ for all $x \in X$ and

$$
P_{t}^{C}\left(x_{A}, x_{B}\right)=\hat{P}_{t}^{C}\left(u_{t}\left(x_{A}\right) / u_{t}\left(x_{B}\right)\right) .
$$

An example would be the Multiplicative Bias Model, in which each type $t$ voter draws bias $\beta$ and votes for $A$ if $u_{t}\left(x_{A}\right)>\beta u_{t}\left(x_{B}\right)$, votes for $B$ if the inequality is reversed, and flips a coin in the event of equality. When $G_{t}$ is continuous, expected plurality share functions are again positive affine transformations of bias distributions, and assumptions on the former translate directly to assumptions on the latter. In much of the paper, we focus on the Utility Difference Model rather than the Utility Ratio Model, but, in Section 9, we will see that the two models are essentially interchangeable: any result for one can be translated into a corresponding result for the other.

\subsection{Alternative Objective Functions}

Under the expected plurality share interpretation of our model, there exist expected vote share functions $V_{t}^{C}$ such that $P_{t}^{C}\left(x_{A}, x_{B}\right)=V_{t}^{C}\left(x_{A}, x_{B}\right)-V_{t}^{B}\left(x_{A}, x_{B}\right)$ for all $t$. Naturally, these functions satisfy

- $V_{t}^{C}\left(x_{A}, x_{B}\right) \geq 0$

- $V_{t}^{A}\left(x_{A}, x_{B}\right)+V_{t}^{B}\left(x_{A}, x_{B}\right) \leq 1$

${ }^{7}$ Biases need not be independently distributed across voter types. 
for all $t$. An alternative would be to interpret $P_{t}^{C}$ as expected vote share, equating $P_{t}^{C}=V_{t}^{C}$, in which case our maintained assumption that $P_{t}^{A}+P_{t}^{B}=0$ is violated.

We stick with the expected plurality share interpretation for two reasons. First, when voters cannot abstain from voting, i.e., $V_{t}^{A}+V_{t}^{B}=1$ for all $t$, the two formulations are equivalent: expected vote share and expected plurality share are positive affine transformations of each other. Second, when abstention is possible, expected vote share maximization can lead to paradoxical conclusions. For example, suppose three voters have single-peaked preferences on the real line, with ideal points 0,1 , and 2 ; if one candidate's platform is preferred by a voter to the other's, the voter votes for the former with probability one; if indifferent, a voter votes for $A$ with probability $1 / 4$, for $B$ with probability $1 / 4$, and abstains with probability $1 / 2$. Then, assuming expected vote maximization, locating at the median is not an equilibrium of the electoral game: candidate $A$ 's expected vote share is increased from $1 / 4$ to $1 / 3$ by moving away from the median, though this move guarantees a loss for $A$.

Of perhaps greater concern, our assumption that payoffs are additive across voter types implies that we cannot generally capture probability of winning maximization directly as a special case of our model. If there is a continuum of voters of each type, however, then we interpret $P_{t}^{A}\left(x_{A}, x_{B}\right)$ as the plurality share actually received by candidate $A$, so the probability $A$ wins is deterministic. Assuming a plurality rule election, it is one if $\int P_{t}^{A} d \mu>0$, zero if the reverse inequality holds, and one half otherwise. Clearly, every pure strategy equilibrium under expected plurality maximization is an equilibrium under probability of winning maximization. The converse may not hold because, under the latter hypothesis, it is possible that a candidate who cannot win picks a platform that does not maximize his/her expected plurality. This disparity is precluded if a minimal symmetry condition is imposed: given any platform for one candidate, the other can choose some platform that at least equalizes their expected plurality shares. This assumption holds, for example, if the voters "flip fair coins" when indifferent between the two candidates.

It follows that if the electorate is a continuum, if the symmetry condition holds, and if voters cannot abstain, then the three objectives coincide, at least in regard to pure strategy equilibria. It appears the electoral game with expected plurality maximizing candidates may have different mixed strategy equilibria than the game with probability of winning candidates in a plurality rule election. Moreover, if the electorate is finite, then our additive formulation of payoffs may lead to different pure strategy equilibria than the probability of winning formulation.

\section{Existence and Uniqueness}

In this section, we take up the issues of existence and uniqueness of equilibria in the electoral game. Hinich, Ledyard, and Ordeshook $(1972,1973)$ first noted that, when uncertainty is added to the canonical spatial model of elections, the electoral game can have pure strategy equilibria, unique under certain conditions. Subsequent results have typically strengthened their original assumptions, yielding sharper characterizations of equilibrium, a topic to which we return in later sections. In this section, we first establish the existence of mixed 
strategy equilibria, an issue often overlooked, under minimal conditions of compactness and continuity. We then take up the existence of pure strategy equilibria and find that concavity of the candidates' objective functions is a key condition. Strengthening this to strict concavity, we derive uniqueness as well. While our main results are stated for the general model, we close the section with some implications for the Fixed Utility and Utility Difference Models.

Theorem 1 Assume (i) $X$ is compact and (ii) for each $t$ and $C, P_{t}^{C}\left(x_{A}, x_{B}\right)$ is jointly continuous in $\left(x_{A}, x_{B}\right)$. Then there exists a mixed strategy electoral equilibrium of the electoral game.

Proof: The space of mixed strategies for the candidates is convex and is compact and metrizable in the weak* topology. We can apply Glicksberg's (1952) fixed point theorem to deduce the existence of an equilibrium if the candidates' objective functions are jointly continuous: that is, as long as $\left(x_{A}^{m}, x_{B}^{m}\right) \rightarrow\left(x_{A}, x_{B}\right)$ implies

$$
\int_{T} P_{t}^{C}\left(x_{A}^{m}, x_{B}^{m}\right) \mu(d t) \rightarrow \int_{T} P_{t}^{C}\left(x_{A}, x_{B}\right) \mu(d t) .
$$

Assumption (ii) implies that, for each $t, P_{t}^{C}\left(x_{A}^{m}, x_{B}^{m}\right)$ converges to $P_{t}^{C}\left(x_{A}, x_{B}\right)$. By our background assumptions on expected plurality share functions, we know the sequence $\left\{P_{(\cdot)}^{C}\left(x_{A}^{m}, x_{B}^{m}\right)\right\}$ is bounded. Therefore, by Lebesgue's dominated convergence theorem, the integrals converge as desired.

An application to the Fixed Utility Model is clear: there, $P_{t}^{C}\left(x_{A}, x_{B}\right)=\tilde{P}_{t}^{C}\left(u_{t}\left(x_{A}\right)\right.$, $\left.u_{t}\left(x_{B}\right)\right)$, so continuity of $u_{t}$ and $\tilde{P}_{t}^{C}$ yields continuity of $P_{t}^{C}$; with compactness of $X$, a mixed strategy equilibrium exists.

Hinich, Ledyard, and Ordeshook allow for "non-rectangular" sets of strategy profiles, incorporating restrictions such as one candidate cannot locate to the left of the other on a particular dimension. ${ }^{8}$ Moreover, Hinich, Ledyard, and Ordeshook prove uniqueness under the expected vote share interpretation of the model, which creates a non-constant sum game when abstention is allowed. We do not take up these issues here, and our analysis is dramatically simplified as a consequence: whereas Hinich, Ledyard, and Ordeshook rely on an existence and uniqueness result for "concave games" due to Rosen (1965), our arguments employ elementary results of game theory. ${ }^{9}$

Before examining uniqueness, we first verify well-known sufficient conditions for existence of pure strategy equilibrium.

Theorem 2 Assume (i) $X$ is compact and convex, (ii) for each $t$ and $C, P_{t}^{C}\left(x_{A}, x_{B}\right)$ is jointly continuous in $\left(x_{A}, x_{B}\right)$, (iii) for each $x_{A}$ and $x_{B}, \int P_{t}^{A}\left(x_{A}, x_{B}\right) d \mu$ is quasi-concave in $x_{A}$ and $\int P_{t}^{B}\left(x_{A}, x_{B}\right) d \mu$ is quasi-concave in $x_{B}$. Then there exists a pure strategy electoral equilibrium.

\footnotetext{
${ }^{8}$ This kind of restriction may be viewed as a reduced form of a more complex model in which candidates can feasibly locate themselves independently but, due to the structure of payoffs, would never find it profitable to do so.

${ }^{9}$ In Theorem 2 we could have allowed for any compact, convex set of feasible strategy profiles, thereby allowing for non-rectangularities, by appealing to the theorem of Banks and Duggan (2001).
} 
Proof: Continuity of the candidates' objective functions follows as in the proof of Theorem 1. Since the objective functions are quasi-concave, Glickberg's (1952) theorem can be applied in the space of platform pairs to deliver a pure strategy equilibrium.

Of course, since the integral of concave functions is concave, a sufficient condition for (iii) in Theorem 2 is that, for each $t$ and $x_{B}, P_{t}^{A}\left(x_{A}, x_{B}\right)$ is concave in $x_{A}$. In fact, this condition is sufficient for concavity, rather than merely quasi-concavity, of the candidates' objective functions in their own strategies. A notable implication of this stronger condition is that, since expected plurality shares lie between zero and one, the policy space is either bounded or the candidates' objective functions are actually constant in their own strategies. We do impose compactness in Theorem 2, however, so the assumption of concavity would not imply the objective functions are constant.

Strengthening conditions (iii) and (iv) of Theorem 2, we can establish uniqueness as well. The next result is closely related to the existence and uniqueness part of Lindbeck and Weibull's (1993) Theorem 1, though we strengthen their strict quasi-concavity to strict concavity in order to capture mixed strategy electoral equilibria. We give further uniqueness results in Section 4.

Theorem 3 Assume (i) $X$ is compact and convex, (ii) for each $t$ and $C, P_{t}^{C}\left(x_{A}, x_{B}\right)$ is jointly continuous in $\left(x_{A}, x_{B}\right)$, (iii) for each $x_{A}$ and $x_{B}, \int P_{t}^{A}\left(x_{A}, x_{B}\right) d \mu$ is strictly concave in $x_{A}$ and $\int P_{t}^{B}\left(x_{A}, x_{B}\right) d \mu$ is strictly concave in $x_{B}$. Then there is exactly one electoral equilibrium, and it is in pure strategies.

Proof: Existence of equilibrium follows from Theorems 1 or 2. By (iii), candidate $A$ 's expected payoff given a mixed strategy for $B$,

$$
\int_{X} \int_{T} P_{t}^{A}\left(x_{A}, x_{B}\right) \mu(d t) \pi_{B}\left(d x_{B}\right),
$$

is strictly concave in $x_{A}$, and likewise for $B$. Thus, all best responses to mixed strategies, and therefore all electoral equilibria, are in pure strategies. If there are distinct equilibria, say $\left(x_{A}^{\prime}, x_{B}^{\prime}\right)$ and $\left(x_{A}^{\prime \prime}, x_{B}^{\prime \prime}\right)$, we can suppose without loss of generality that $x_{A}^{\prime} \neq x_{A}^{\prime \prime}$. The electoral game is constant sum, and therefore its equilibria are interchangeable. Thus, $\left(x_{A}^{\prime \prime}, x_{B}^{\prime}\right)$ is an equilibrium, implying that $x_{A}^{\prime}$ and $x_{A}^{\prime \prime}$ are distinct best responses to $x_{B}^{\prime}$. By strict concavity, however, $\frac{1}{2} x_{A}^{\prime}+\frac{1}{2} x_{A}^{\prime \prime}$ yields a higher payoff to candidate $A$, a contradiction.

The application of Theorems 2 and 3 to the Fixed Utility Model is straightforward, after establishing the following simple lemma. This gives us essentially Hinich, Ledyard, and Ordeshook's (1973) Theorem 1. Note that we can assume only aggregate strict concavity, rather than assuming some types of voters have strictly concave utility functions.

Lemma 1 In the Fixed Utility Model, assume (i) for eacht and $C, \tilde{P}_{t}^{C}$ is weakly monotonic, (ii) for each $t$ and $v, \tilde{P}_{t}^{A}(u, v)$ is concave in $u$ on $\mathcal{U}_{t}$, (iii) for each $t$ and $u, \tilde{P}_{t}^{B}(u, v)$ is concave in $v$ on $\mathcal{U}_{t}$, and (iv) for each $t, u_{t}$ is concave. Then for each $x_{A}$ and $x_{B}$, $\int P_{t}^{A}\left(x_{A}, x_{B}\right) d \mu$ is concave in $x_{A}$ and $\int P_{t}^{B}\left(x_{A}, x_{B}\right) d \mu$ is concave in $x_{B}$. 
Assume, moreover, (v) for each $t$ and $C, \tilde{P}_{t}^{C}$ is strictly monotonic, and (vi) aggregate strict concavity holds. Then for each $x_{A}$ and $x_{B}, \int P_{t}^{A}\left(x_{A}, x_{B}\right) d \mu$ is strictly concave in $x_{A}$ and $\int P_{t}^{B}\left(x_{A}, x_{B}\right) d \mu$ is strictly concave in $x_{B}$.

Proof: We first check that, for all $v, \int \tilde{P}_{t}^{A}\left(u_{t}\left(x_{A}\right), v\right) d \mu$ is concave in $x_{A}$. Take any distinct $x_{A}^{\prime}, x_{A}^{\prime \prime}$ and any $\alpha \in(0,1)$. Then, for all $t$,

$$
\begin{aligned}
\tilde{P}_{t}^{A}\left(u_{t}\left(\alpha x_{A}^{\prime}+(1-\alpha) x_{A}^{\prime \prime}\right), v\right) & \geq \tilde{P}_{t}^{A}\left(\alpha u_{t}\left(x_{A}^{\prime}\right)+(1-\alpha) u_{t}\left(x_{A}^{\prime \prime}\right), v\right) \\
& \geq \alpha \tilde{P}_{t}^{A}\left(u_{t}\left(x_{A}^{\prime}\right), v\right)+(1-\alpha) \tilde{P}_{t}^{A}\left(u_{t}\left(x_{A}^{\prime \prime}\right), v\right),
\end{aligned}
$$

where the first equality follows from (i) and (iv), the second from (ii). Since the integral of concave functions is concave, $A$ 's objective function is concave in $x_{A}$. By (v) and (vi), the first equality holds strictly for a set of types with positive measure, implying that $A$ 's objective function is strictly concave in $x_{A}$. A symmetric argument applies for $B$.

We use Lemma 1 to provide two applications of the above results to the Utility Difference Model. ${ }^{10}$

Corollary 1 In the Utility Difference Model, assume (i) $X$ is compact and convex, (ii) for each $t$ and $C, \hat{P}_{t}^{C}$ is continuous and weakly monotonic, (iii) for all $t, \hat{P}_{t}^{A}(u)$ is concave on $\mathcal{U}_{t}-\mathcal{U}_{t}$, (iv) for all $t, \hat{P}_{t}^{B}(-u)$ is concave on $\mathcal{U}_{t}-\mathcal{U}_{t}$, and $(v)$ for each $t, u_{t}$ is continuous and concave. Then there exists a pure strategy electoral equilibrium.

Assume, moreover, (vi) for each $t$ and $C, \hat{P}_{t}^{C}$ is strictly monotonic, and (vii) aggregate strict concavity holds. Then there is exactly one electoral equilibrium, and it is in pure strategies.

If nothing more than concavity is known of voter utility functions, then concavity of $\hat{P}_{t}^{A}(u)$ and $\hat{P}_{t}^{B}(-u)$ is needed to guarantee concavity of the candidates' objective functions in Corollary 1. But this has the strong implication that $\hat{P}_{t}^{A}$ and $\hat{P}_{t}^{B}$ are affine linear on $\mathcal{U}_{t}-\mathcal{U}_{t}$. Thus, Corollary 1 applies to the Additive Bias Model as long as the bias term is uniformly distributed over a wide enough range, namely, a range including the set $\mathcal{U}_{t}-\mathcal{U}_{t}$ of possible Utility Differences. As we show in Section 4, however, we can prove much more than the existence and uniqueness result of Corollary 1 when we know the expected plurality share functions of the candidates are affine linear. Namely, we can show that pure strategy equilibrium platforms must maximize a utilitarian welfare function, and that these platforms are actually dominant strategies for the candidates.

If we strengthen concavity of the voters' utility functions, however, then we can weaken concavity of the expected plurality share functions, allowing for different distributions of the bias term in the Additive Bias Model. The next corollary does just this, and it anticipates our discussion, in Section 9, on the connections between the Utility Difference and Ratio Models.

\footnotetext{
${ }^{10}$ In fact, Lindbeck and Weibull's (1993) argument in the proof of their Theorem 1 establishes that the unique electoral equilibrium of the second part of Corollaries 1 and 2 must exhibit policy coincidence. We give more general results on policy coincidence in Section 5.
} 
Corollary 2 In the Utility Difference Model, assume (i) $X$ is compact and convex, (ii) for each $t$ and $C, \hat{P}_{t}^{C}(\ln (u))$ is continuous and weakly monotonic in $u$, (iii) for each $t$, $\hat{P}_{t}^{A}(\ln (u))$ is concave on $\mathcal{U}_{t}-\mathcal{U}_{t}$, (iv) for each $t, \hat{P}_{t}^{B}(-\ln (u))$ is concave on $\mathcal{U}_{t}-\mathcal{U}_{t}$, and $(v)$ for each $t, u_{t}$ is continuous and $e^{u_{t}}$ is concave. Then there exists a pure strategy electoral equilibrium.

Assume, moreover, (vi) for each $t$ and $C, \hat{P}_{t}^{C}$ is strictly monotonic, and (vii) aggregate strict concavity holds with respect to the exponentials of voter utility functions. Then there is exactly one electoral equilibrium, and it is in pure strategies.

The proof follows easily upon verifying the assumptions of Theorems 2 and 3. For an example of expected plurality share functions in the Utility Difference Model satisfying (ii)-(iv), (vi), and (vii) from Corollary 2 but not concavity, consider

$$
\hat{P}_{t}^{A}(u)=\frac{1-e^{-u}}{1+e^{-u}} \quad \text { and } \quad \hat{P}_{t}^{B}(u)=\frac{e^{-u}-1}{1+e^{-u}} .
$$

Ultimately, of course, the minimal condition on expected plurality shares and utility functions needed for existence of pure strategy equilibrium is just: for all $x_{B}, \hat{P}_{t}^{A}\left(u_{t}\left(x_{A}\right)-\right.$ $u_{t}\left(x_{B}\right)$ ) concave in $x_{A}$ (and similarly for $B$ ). Corollaries 1 and 2 just serve to break this into separate conditions on voter utility functions and the expected plurality share functions $\hat{P}_{t}^{C}$ in the Utility Difference Model. See Lindbeck and Weibull's (1987) Theorem 2 for a joint sufficient condition on the two primitives in their distributive setting.

\section{Social Welfare Optima}

In this section, we establish a correspondence between pure strategy equilibria of the electoral game and optima of a utilitarian social welfare function: under very general conditions, pure strategy equilibrium platforms must maximize welfare. Indeed, our most general necessity result is stated in the Fixed Utility Model and assumes only differentiability of expected plurality share functions and concavity of voter utility functions. This result is in the same spirit as Ledyard's (1984) utilitarian result, proved in a different framework, and extends results by Lindbeck and Weibull $(1987,1993)$ and Coughlin (1992). In fact, we can drop concavity of voter utility functions and, when the expected plurality share functions are linear, obtain a strong equivalence between utilitarian optima and pure strategy equilibria. Finally, using our utilitarian theorems, we derive Hinich's $(1977,1978)$ result on the mean voter, a topic we return to in Section 8.

Regarding their implications for social welfare, the theorems of this section do yield Pareto optimality of the candidates' equilibrium platforms. Because they are stated in terms of utilitarian social welfare functions, the results may seem to suggest even more: the selection of a normatively superior Pareto optimal policy. It is well-known, however, that such a selection requires interpersonal comparisons of utility and raises a prior problem: the selection of "normatively superior" welfare weights and utility representations of voter preferences. The welfare weights defined in the theorems below are given by each voter type's marginal expected pluralities in favor of one candidate or the other, and these would appear to have no special normative basis. Utilities can be compared across voters in the 
Fixed Utility Model because the voter utility functions selected there contain information about probabilities of voting for one candidate or the other - and these probabilities can be compared. Though that selection of utility representations is technically convenient and even natural, however, there is no presumption of its normative priority.

\subsection{Characterization Results}

We first use strong restrictions on expected plurality share functions, namely that they are linear, to show that maximizing social welfare is necessary and sufficient for platforms to be used in equilibrium. Because we do not need to assume concavity of the candidates' objective functions (or, specifically, the voters' utility functions), this gives alternative conditions to those of Theorem 2 for existence, and it gives alternative conditions to those of Theorem 3 for uniqueness.

Theorem 4 In the Fixed Utility Model, assume that for each $t$ and all $u, v \in \mathcal{U}_{t}, \tilde{P}_{t}^{A}(u, v)$ is affine linear in $u$ with coefficient $a_{t}>0$ (independent of $v$ ), and $\tilde{P}_{t}^{B}(u, v)$ is affine linear in $v$ with coefficient $b_{t}>0$ (independent of $u$ ). Then $\left(\pi_{A}^{*}, \pi_{B}^{*}\right)$ is a mixed strategy electoral equilibrium if and only if $\pi_{A}^{*}$ and $\pi_{B}^{*}$ put probability one on maximizers of the weighted utilitarian social welfare functions

$$
\int_{T} a_{t} u_{t}(x) \mu(d t) \text { and } \int_{T} b_{t} u_{t}(x) \mu(d t),
$$

respectively. If $a_{t}$ and $b_{t}$ are independent of $t$, then $\pi_{A}^{*}$ and $\pi_{B}^{*}$ both put probability one on the maximizers of the utilitarian social welfare function

$$
\int_{T} u_{t}(x) \mu(d t)
$$

If the welfare functions have unique maximizers, say $\tilde{x}_{A}$ and $\tilde{x}_{B}$, then $\left(\tilde{x}_{A}, \tilde{x}_{B}\right)$ is the unique electoral equilibrium.

Proof: Note that $x_{A}$ is a best response to $\pi_{B}^{*}$ for $A$ if and only if $x_{A}^{*}$ maximizes

$$
\int_{T} \tilde{P}_{t}^{A}\left(u_{t}(x), u_{t}\left(x_{B}^{*}\right)\right) \mu(d t)=\int_{T}\left[a_{t} u_{t}(x)+c_{t}\right] \mu(d t),
$$

which is a positive affine transformation of $A$ 's welfare function. A similar argument for $B$ shows that maximizing the welfare function is necessary and sufficient for an electoral equilibrium. The last claims of the theorem are self-evident.

As is clear from the proof, under the conditions of Theorem 4, the welfare maximizing platforms are actually dominant strategies for the candidates, in the sense that those platforms are best responses for a candidate regardless of the other candidate's platforms. Furthermore, in the special case of the Utility Difference Model with linear $\hat{P}_{t}^{C}$, we have

$$
\begin{aligned}
& \tilde{P}_{t}^{A}(u, v)=\hat{P}_{t}^{A}(u-v)=a_{t}(u-v)+c_{t} \\
& \quad=-b_{t}(u-v)+d_{t}=\hat{P}_{t}^{B}(u-v)=\tilde{P}_{t}^{B}(u, v),
\end{aligned}
$$


so that the coefficient $a_{t}$ is automatically independent of $v$ (and $b_{t}$ is independent of $u$ ), and $a_{t}=b_{t}$ for all $t$. Thus, in any electoral equilibrium of the Additive Bias Model with a bias term uniformly distributed over a wide enough range, the candidates maximize the same weighted utilitarian welfare function.

We can derive much more general necessary conditions for pure strategy equilibria to solve social welfare maximization problems. The next theorem generalizes the first order analysis of Theorem 1 of Lindbeck and Weibull (1987), who consider the distributive setting. With Theorem 3, it extends Lindbeck and Weibull's (1993) Theorem 1, which includes sufficient conditions for existence, to mixed strategy equilibria in the multidimensional setting. It also generalizes half of Coughlin's (1992) Corollary 4.4, proved in connection to his results on Nash social welfare, a connection we develop in Section 9. In contrast to our theorem, Coughlin's utilitarian result is stated as a necessary and sufficient condition for pure strategy equilibrium, because he imposes the Binary Luce functional form, fulfilling the sufficient conditions for existence in Theorem 2 .

Theorem 5 In the Fixed Utility Model, assume (i) for each $t$ and $C, \tilde{P}_{t}^{C}(u, v)$ is continuously differentiable in $(u, v)$, (ii) for each $C$, the partial derivatives of $\tilde{P}_{t}^{C}(u, v)$ are bounded in $(t, u, v)$, and (iii) for each $t, u_{t}(x)$ is continuously differentiable on the interior of $X$ with derivatives bounded in $(t, x)$. If $\left(x_{A}^{*}, x_{B}^{*}\right)$ is an interior pure strategy electoral equilibrium, then $x_{A}^{*}$ is a critical point of the weighted utilitarian social welfare function

$$
\int_{T} \alpha_{t} u_{t}(x) \mu(d t)
$$

where $\alpha_{t}=D_{u=u_{t}\left(x_{A}^{*}\right)} \tilde{P}_{t}^{A}\left(u, u_{t}\left(x_{B}^{*}\right)\right)$ for all $t$, and $x_{B}^{*}$ is a critical point of the weighted utilitarian social welfare function

$$
\int_{T} \beta_{t} u_{t}(x) \mu(d t)
$$

where $\beta_{t}=D_{v=u_{t}\left(x_{B}^{*}\right)} \tilde{P}_{t}^{B}\left(u_{t}\left(x_{A}^{*}\right), v\right)$ for all $t$.

Assume, moreover, (iv) $\alpha_{t}$ and $\beta_{t}$ are positive and independent of $t$. Then $x_{A}^{*}$ and $x_{B}^{*}$ are critical points of

$$
\int_{T} u_{t}(x) \mu(d t)
$$

Assume, moreover, (v) $X$ is convex and (vi) aggregate strict concavity holds. Then $x_{A}^{*}=x_{B}^{*}$ is the unique maximizer of (3).

Proof: If $\left(x_{A}^{*}, x_{B}^{*}\right)$ is an interior equilibrium, then $x_{A}^{*}$ solves

$$
\max _{x_{A} \in X} \int_{T} \tilde{P}_{t}^{A}\left(u_{t}\left(x_{A}\right), u_{t}\left(x_{B}^{*}\right)\right) \mu(d t) .
$$

By (i) and (ii), and since $\left(x_{A}^{*}, x_{B}^{*}\right)$ is interior, the above integral is differentiable at $\left(x_{A}^{*}, x_{B}^{*}\right)$, and the necessary first order condition for a maximum holds:

$$
D_{x_{A}=x_{A}^{*}} \int_{T} \tilde{P}_{t}^{A}\left(u_{t}\left(x_{A}\right), u_{t}\left(x_{B}^{*}\right)\right) \mu(d t)=0 .
$$


Using (i)-(iii), we interchange differentiation and integration and use the chain rule to obtain

$$
\int_{T} D_{u=u_{t}\left(x_{A}^{*}\right)} \tilde{P}_{t}^{A}\left(u, u_{t}\left(x_{B}^{*}\right)\right) D_{x=x_{A}^{*}} u_{t}(x) \mu(d t)=0
$$

which implies

$$
\int_{T} \alpha_{t} D_{x=x_{A}^{*}} u_{t}(x) \mu(d t)=0
$$

Therefore, $x_{A}^{*}$ is a critical point of $\int \alpha_{t} u_{t}(x) d \mu$, with a similar argument applying for $B$. Assuming (iv), let $\alpha=\alpha_{t}>0$ for all $t$. Dividing both sides of (4) by $\alpha$, we see that $x_{A}^{*}$ is a critical point of (3), and similarly for $B$. Adding (v) and (vi), $\int u_{t}(x) d \mu$ is strictly concave, and the last claim of the theorem is self-evident.

As is clear from the proof, the necessary conditions given in the first parts of Theorem 5 actually hold for all interior local electoral equilibria, which requires merely that each candidate's platform maximize the candidate's expected plurality share over some open set containing the platform. The result can be proved without imposing differentiability conditions on voter utility functions, and without assuming interiority of equilibrium, if we add some convex structure to the problem. Next, we impose concavity of voter utility functions at the outset to prove the utilitarian result.

Theorem 6 In the Utility Difference Model, assume (i) $X$ is convex, (ii) for each $t$ and $C$, $\tilde{P}_{t}^{C}(u, v)$ is strictly monotonic and has partial derivatives with respect to the $C$ th component bounded in $(t, u, v)$, and (iii) for each $t, u_{t}$ is concave. If $\left(x_{A}^{*}, x_{B}^{*}\right)$ is a pure strategy electoral equilibrium, then $x_{A}^{*}$ maximizes (1) and $x_{B}^{*}$ maximizes (2).

Assume, moreover, (iv) $\alpha_{t}$ and $\beta_{t}$ are positive and independent of $t$. Then $x_{A}^{*}$ and $x_{B}^{*}$ maximize (3).

Assume, moreover, (v) aggregate strict concavity holds. Then $x_{A}^{*}=x_{B}^{*}$ is the unique maximizer of (3).

Proof: We first prove the result assuming $T$ is finite. Suppose $\left(x_{A}^{*}, x_{B}^{*}\right)$ is an equilibrium but $x_{A}^{*}$ does not maximize (1), so there exists $x^{\prime} \in X$ such that

$$
\sum_{T} \alpha_{t} u_{t}\left(x^{\prime}\right) \mu(\{t\})>\sum_{T} \alpha_{t} u_{t}\left(x_{A}^{*}\right) \mu(\{t\}) .
$$

Let $P: \Re^{T} \rightarrow \Re$ be the function defined by

$$
P(z)=\sum_{t \in T} P_{t}^{A}\left(z_{t}, u_{t}\left(x_{B}^{*}\right)\right) \mu(\{t\})
$$

for all $z=\left(z_{t}\right)_{t \in T} \in \Re^{T}$, and let

$$
\mathcal{U}=\left\{z \in \Re^{T} \mid \exists x \in X, \forall t \in T, z_{t} \leq u_{t}(x)\right\},
$$


a convex set by (i) and (iii). By the assumption that $\left(x_{A}^{*}, x_{B}^{*}\right)$ is an equilibrium, $z^{*}=$ $\left(u_{t}\left(x_{A}^{*}\right)\right)_{t \in T}$ solves

$$
\begin{gathered}
\max _{z \in \Re^{T}} P(z) \\
\text { s.t. } z \in \mathcal{U} .
\end{gathered}
$$

By (ii), $P$ is differentiable at $z^{*}$, and

$$
D_{z=z^{*}} P(z)=\left(\alpha_{t_{1}} \mu\left(\left\{t_{1}\right\}\right), \ldots, \alpha_{t_{K}} \mu\left(\left\{t_{K}\right\}\right)\right),
$$

where $\alpha_{t}$ is defined as in Theorem 5 , and where $T=\left\{t_{1}, \ldots, t_{K}\right\}$. Now let $z^{\prime}=\left(u_{t}\left(x^{\prime}\right)\right)_{t \in T}$, and note that

$$
D_{z=z^{*}} P(z) \cdot\left(z^{\prime}-z^{*}\right)=\sum_{t \in T} \alpha_{t}\left[u_{t}\left(x^{\prime}\right)-u_{t}\left(x^{*}\right)\right] \mu(\{t\})>0 .
$$

Thus, $D_{z=z^{*}} P(z) \cdot \alpha\left(z^{\prime}-z^{*}\right)>0$ for all $\alpha>0$. Taking $\alpha>0$ small enough, we therefore have $z^{*}+\alpha\left(z^{\prime}-z^{*}\right) \in \mathcal{U}$ and $P\left(z^{*}+\alpha\left(z^{\prime}-z^{*}\right)\right)>P\left(z^{*}\right)$, a contradiction. Therefore, $x_{A}^{*}$ maximizes (1).

When $T$ is infinite, the proof proceeds exactly as above, but some concepts must be extended to the general case. Integrals replace summation signs, and $P$ may be defined on the subspace of $\Re^{T}$ consisting of the bounded, measurable functions of $t$, endowed with the sup norm. Thus, $P$ is a "functional." If we use the concept of Fréchet derivative (see Luenberger (1969)), then $P$ is differentiable at $z^{*}$ under our assumptions, and

$$
D_{z=z^{*}} P(z)\left(z^{\prime}-z^{*}\right)=\int_{T} \alpha_{t}\left[u_{t}\left(x^{\prime}\right)-u_{t}\left(x^{*}\right)\right] \mu(d t)>0,
$$

as above, again resulting in a contradiction. Of course, the same argument applies to $x_{B}^{*}$. The remainder of the theorem is self-evident.

Theorem 6 has an easy implication, stated next without proof, for pure strategy electoral equilibria exhibiting policy coincidence in the Utility Difference Model. We have already established conditions under which such equilibria exist. In the next section, we give several sets of conditions under which pure strategy equilibria of the electoral game necessarily exhibit policy coincidence.

Corollary 3 In the Utility Difference Model, assume (i) $X$ is convex, (ii) for each $t$ and $C, \hat{P}_{t}^{C}(u)$ is strictly monotonic and differentiable with derivatives bounded in $(t, u)$ and with $D_{u=0} \hat{P}_{t}^{C}(u)$ independent of $t$, and (iii) for each $t, u_{t}(x)$ is concave. If $\left(x^{*}, x^{*}\right)$ is an interior pure strategy electoral equilibrium, then $x^{*}$ maximizes (3).

Assume, moreover, (iv) aggregate strict concavity holds. Then $\left(x^{*}, x^{*}\right)$ is the unique interior pure strategy electoral equilibrium exhibiting policy coincidence.

The assumptions in Corollary 3 isolate one case in which the welfare weights of the different voter types are equal. The key condition used there is that the marginal expected plurality shares of the candidates are equal across voter types when all voter types are indifferent between the two candidates, a condition satisfied if expected plurality share 
functions are symmetric across voter types. The theorem has two interesting connotations. First, if we view voter utility functions as valuations in terms of a quasi-linear private good, then it is well-known that Pareto optimality is equivalent to maximizing the unweighted sum of voter utilities. Under the assumptions of the theorem, therefore, pure strategy equilibria exhibiting policy coincidence are Pareto optimal. But, if voter preferences are quasi-linear and the marginal expected plurality shares of the candidates are not equal across voter types when indifferent toward the platforms of the candidates, equilibrium outcomes may not be Pareto optimal. Second, under the conditions of the theorem, the pure strategy equilibria exhibiting policy coincidence, if they exist, are independent of the candidates' expected plurality share functions and, therefore, independent of the structure of incomplete information in the model.

\subsection{The Mean Voter Theorem}

We say preferences are generalized Euclidean if (a) for each $t$, there exists $x^{t} \in X$ and an $n \times n$ matrix $A_{t}=\left(a_{i j}^{t}\right)$ such that

$$
u_{t}(x)=-\left(x-x^{t}\right) A_{t}\left(x-x^{t}\right),
$$

for all $x \in X$, (b) $x^{t}$ and $A_{t}$ are bounded and measurable with respect to $t$, and (c) letting $A_{t}^{\prime}$ denote the transpose of $A_{t}, \int\left[A_{t}+A_{t}^{\prime}\right] d \mu$ is invertible. A special case is quadratic preferences, where $A_{t}=I, x^{t}$ is the ideal point of type $t$ voters, and $u_{t}(x)=-\left\|x-x^{t}\right\|^{2}$. We next derive half of Hinich's (1978) Theorem 1 on the "mean voter" from our utilitarian results. His theorem is stronger than our result in one respect: whereas we assume policy coincidence, Hinich derives it. In the next section, we take up this issue in full detail.

Corollary 4 In the Utility Difference Model, assume (i) for each t and $C, \hat{P}_{t}^{C}(u)$ is strictly monotonic and differentiable with derivatives bounded in $(t, u)$ and with $D_{u=0} \hat{P}_{t}^{C}(u)$ independent of $t$, and (ii) preferences are generalized Euclidean. If $\left(x^{*}, x^{*}\right)$ is an interior pure strategy electoral equilibrium, then

$$
x^{*}=\left(\int_{T}\left[A_{t}+A_{t}^{\prime}\right] \mu(d t)\right)^{-1}\left(\int_{T}\left[A_{t}+A_{t}^{\prime}\right] x^{t} \mu(d t)\right) .
$$

Assume, moreover, (iv) $A_{t}$ is symmetric and independent of $t$, then

$$
x^{*}=\int_{T} x^{t} \mu(d t) .
$$

Proof: From Corollary 3, we know $x^{*}$ is a critical point of $\int u_{t}(x) d \mu$. Thus, $\int D_{x=x^{*}} u_{t}(x) d \mu=$ 0 . Note that, for each $t$,

$$
\begin{aligned}
u_{t}(x) & =-\sum_{i=1}^{n} \sum_{j=1}^{n} a_{i j}^{t}\left(x_{i}-x_{i}^{t}\right)\left(x_{j}-x_{j}^{t}\right) \\
\frac{\partial u_{t}}{\partial x_{k}}(x) & =-\sum_{i=1}^{n}\left(a_{i k}^{t}+a_{k i}^{t}\right)\left(x_{i}-x_{i}^{t}\right) \\
D u_{t}(x) & =-\left[A_{t}+A_{t}^{\prime}\right]\left(x-x^{t}\right) .
\end{aligned}
$$


Thus, the first order condition reduces to

$$
\int_{T}\left[A_{t}+A_{t}^{\prime}\right]\left(x^{*}-x^{t}\right) \mu(d t)=0,
$$

which gives us (5). Assuming (iv), $x^{*}$ is the mean ideal point, as claimed.

The mean voter result also appeared in Hinich's (1977) 'Three Voter Example,' and earlier in Hinich, Ledyard, and Ordeshook's (1973) Theorems 5 and 6. Their Theorem 5 differs from our Corollary 4 in that it restricts the distribution of voter ideal points (imposing symmetry) rather than any single voter's utility function; they do, however, also assume that the utility functions of voters with diametrically opposed ideal points are "mirror images" of each other. Though we do not formalize this claim here, their result can also be derived from Theorem 5: under their conditions, the mean (also mode) of the voters' ideal points maximizes the utilitarian social welfare function. Hinich, Ledyard, and Ordeshook's Theorem 6 assumes the candidates' expected plurality share functions are linear in platforms adopted: in the Fixed Utility Model, this amounts to assuming linear expected plurality share functions (as functions of utility levels) and generalized Euclidean voter utility functions.

\subsection{A Further Uniqueness Result}

Corollary 3, and Theorems 5 and 6 more generally, give conditions under which there is at most one interior pure strategy electoral equilibrium exhibiting policy coincidence. We end this section by adding a uniqueness result based on this property. The result rests on the familiar property of interchangeability of equilibria for two-player zero-sum games and holds in the general electoral model.

Theorem 7 Assume (i) for each $t, C$, and $(x, y), P_{t}^{A}(x, y)=P_{t}^{B}(y, x)$, and (ii) $\left(x^{*}, x^{*}\right)$ is the unique (interior) pure strategy electoral equilibrium exhibiting policy coincidence. If $\left(x_{A}, x_{B}\right)$ is an (interior) pure strategy electoral equilibrium, then $x_{A}=x_{B}=x^{*}$.

Proof: By (i), the electoral game is a symmetric, two-player, zero-sum game. If $\left(x_{A}, x_{B}\right)$ is an (interior) pure strategy electoral equilibrium, then so is $\left(x_{B}, x_{A}\right)$. By interchangeability, $\left(x_{A}, x_{A}\right)$ is an (interior) pure strategy electoral equilibrium, and by (ii) we have $x_{A}=x^{*}$. Similarly, $x_{B}=x^{*}$.

Thus, under the assumption of symmetry, we can extend the above uniqueness results for electoral equilibria exhibiting policy coincidence to all pure strategy electoral equilibria. Of note, Lindbeck and Weibull's (1987) uniqueness argument (in their Section 4) does not impose symmetry and does not follow from our results: their argument uses the special structure of the distributive model. 


\section{Policy Coincidence}

In this section, we consider more deeply the incentives for candidates to match each other's policy platform, giving voters essentially just one choice of policy. If the expected plurality share functions of the candidates are symmetric, in the sense of condition (i) from Theorem 7 , then, under the conditions of Theorem 2, the existence of symmetric equilibria follows from standard arguments. While we therefore have conditions under which some equilibria exhibit policy coincidence, we now examine whether all equilibria must exhibit policy coincidence, a feature of elections first noted by Hotelling (1929) in the Deterministic Model. Theorem 7, with Corollary 3, currently delivers policy coincidence in the Utility Difference Model under rather weak conditions: chief among them are that the marginal expected plurality shares of the candidates are independent of voter type when voters are indifferent, and that the electoral game is symmetric.

In the existing literature, Hinich, Ledyard, and Ordeshook (1973) give conditions necessitating policy coincidence, ones involving strict concavity of expected plurality share functions. Calvert (1985) weakens strict concavity to the existence of an "estimated median." Coughlin (1992) weakens strict concavity of voter utility functions to strict "log concavity," but he imposes the Binary Luce functional form on $\tilde{P}_{t}^{C} \cdot{ }^{11}$ Furthermore, all of these authors assume symmetry of the electoral game. In fact, all of these results on policy coincidence are proved under assumptions guaranteeing a unique pure strategy electoral equilibrium. But if $\left(x_{A}^{*}, x_{B}^{*}\right)$ is the unique pure strategy equilibrium of the symmetric electoral game, then it is immediate that $x_{A}^{*}=x_{B}^{*}$, for otherwise, $\left(x_{B}^{*}, x_{A}^{*}\right)$ would be a distinct equilibrium.

Is policy coincidence necessitated by the logic of electoral under weaker assumptions? Hinich (1978) gives one such set of conditions: he shows that, in the Additive Bias Model with generalized Euclidean voter utility functions, every pure strategy equilibrium has the candidates adopting the generalized mean and, therefore, exhibits policy coincidence. He assumes equal marginal expected plurality shares across voter types, but he does not assume symmetry (in his notation, $F_{\epsilon}$ need not be symmetric) or concavity of the candidates' objective functions, and indeed there may not be a pure strategy equilibrium. Lindbeck and Weibull's (1987) Theorem 1 establishes that policy coincidence holds generally in the distributive politics model without assuming symmetry or strict concavity of expected plurality share functions, whereas Lindbeck and Weibull (1993) add strict quasi-concavity of expected plurality share functions to obtain this result in a one-dimensional model. In a different framework, Ledyard (1984) uses symmetry to establish policy coincidence, but his result does not rely on concavity of the candidates' objective functions.

We provide general sufficient conditions for policy coincidence in the Utility Difference Model, obtaining the above results on policy coincidence as special cases. We drop the assumption of equal marginal expected plurality shares across voter types; we do not assume candidate symmetry; and we do not impose concavity conditions on expected plurality share functions. We find that, under natural concavity conditions on voter utilities and monotonicity conditions on expected plurality share functions, every pure strategy electoral equilibrium exhibits policy coincidence.

\footnotetext{
${ }^{11}$ Here, we add strictness to log concavity in Coughlin's (1992) Corollary 4.4 to get policy coincidence.
} 
Theorem 8 In the Utility Difference Model, assume (i) $X$ is convex, (ii) for each $t$ and $C$, $\hat{P}_{t}^{C}$ is strictly monotonic, (iii) for each $t, u_{t}$ is concave, and (v) aggregate strict concavity holds. If $\left(x_{A}^{*}, x_{B}^{*}\right)$ is a pure strategy electoral equilibrium, then $x_{A}^{*}=x_{B}^{*}$.

Proof: Suppose $x_{A}^{*} \neq x_{B}^{*}$, and let $y=\frac{1}{2} x_{A}^{*}+\frac{1}{2} x_{B}^{*}$. Because $\left(x_{A}^{*}, x_{B}^{*}\right)$ is an equilibrium, we know that the net gains to $A$ from deviating to $y$ are non-positive:

$$
\int_{T} \hat{P}_{t}^{A}\left(u_{t}(y)-u_{t}\left(x_{B}^{*}\right)\right) \mu(d t) \leq \int_{T} \hat{P}_{t}^{A}\left(u_{t}\left(x_{A}^{*}\right)-u_{t}\left(x_{B}^{*}\right)\right) \mu(d t) .
$$

Define

$$
\begin{aligned}
& T_{A}^{+}=\left\{t \in T \mid u_{t}(y)>u_{t}\left(x_{A}^{*}\right)\right\} \\
& T_{A}^{-}=\left\{t \in T \mid u_{t}(y)<u_{t}\left(x_{A}^{*}\right)\right\}
\end{aligned}
$$

as the sets of types on which $A$ gains and loses, respectively. Since $\hat{P}_{t}^{A}$ is strictly increasing, we have

$$
\begin{aligned}
& t \in T_{A}^{+} \Leftrightarrow \hat{P}_{t}^{A}\left(u_{t}(y)-u_{t}\left(x_{B}^{*}\right)\right)-\hat{P}_{t}^{A}\left(u_{t}\left(x_{A}^{*}\right)-u_{t}\left(x_{B}^{*}\right)\right)>0 \\
& t \in T_{A}^{-} \Leftrightarrow \hat{P}_{t}^{A}\left(u_{t}(y)-u_{t}\left(x_{B}^{*}\right)\right)-\hat{P}_{t}^{A}\left(u_{t}\left(x_{A}^{*}\right)-u_{t}\left(x_{B}^{*}\right)\right)<0 .
\end{aligned}
$$

Thus, we can decompose $A$ 's net gains from deviating to $y$ into gains,

$$
\mathcal{G}_{A}=\int_{T_{A}^{+}}\left[\hat{P}_{t}^{A}\left(u_{t}(y)-u_{t}\left(x_{B}^{*}\right)\right)-\hat{P}_{t}^{A}\left(u_{t}\left(x_{A}^{*}\right)-u_{t}\left(x_{B}^{*}\right)\right)\right] \mu(d t)
$$

and losses

$$
\mathcal{L}_{A}=\int_{T_{A}^{-}}\left[\hat{P}_{t}^{A}\left(u_{t}\left(x_{A}^{*}\right)-u_{t}\left(x_{B}^{*}\right)\right)-\hat{P}_{t}^{A}\left(u_{t}(y)-u_{t}\left(x_{B}^{*}\right)\right)\right] \mu(d t) .
$$

By construction, the integrands of $\mathcal{G}_{A}$ and $\mathcal{L}_{A}$ are positive. Rewriting (6), we have $\mathcal{G}_{A} \leq \mathcal{L}_{A}$.

By the same logic, the net gains to $B$ from deviating to $y$ are non-positive:

$$
\int_{T}\left[-\hat{P}_{t}^{A}\left(u_{t}\left(x_{A}^{*}\right)-u_{t}(y)\right)\right] \mu(d t) \leq \int_{T}\left[-\hat{P}_{t}^{A}\left(u_{t}\left(x_{A}^{*}\right)-u_{t}\left(x_{B}^{*}\right)\right) \mu(d t),\right.
$$

where we use $\hat{P}_{t}^{B}=-\hat{P}_{t}^{A}$. Define

$$
\begin{aligned}
& T_{B}^{+}=\left\{t \in T \mid u_{t}(y)>u_{t}\left(x_{B}^{*}\right)\right\} \\
& T_{B}^{-}=\left\{t \in T \mid u_{t}(y)<u_{t}\left(x_{B}^{*}\right)\right\}
\end{aligned}
$$

as the sets of types on which $B$ gains and loses, respectively. Since $\hat{P}_{t}^{A}$ is strictly increasing, we have

$$
\begin{aligned}
& t \in T_{B}^{+} \Leftrightarrow \hat{P}_{t}^{A}\left(u_{t}\left(x_{A}^{*}\right)-u_{t}\left(x_{B}^{*}\right)\right)-\hat{P}_{t}^{A}\left(u_{t}\left(x_{A}^{*}\right)-u_{t}(y)\right)>0 \\
& t \in T_{B}^{-} \Leftrightarrow \hat{P}_{t}^{A}\left(u_{t}\left(x_{A}^{*}\right)-u_{t}\left(x_{B}^{*}\right)\right)-\hat{P}_{t}^{A}\left(u_{t}\left(x_{A}^{*}\right)-u_{t}(y)\right)<0 .
\end{aligned}
$$

We can therefore decompose $B$ 's net gains from deviating to $y$ into gains

$$
\mathcal{G}_{B}=\int_{T_{B}^{+}}\left[\hat{P}_{t}^{A}\left(u_{t}\left(x_{A}^{*}\right)-u_{t}\left(x_{B}^{*}\right)\right)-\hat{P}_{t}^{A}\left(u_{t}\left(x_{A}^{*}\right)-u_{t}(y)\right)\right] \mu(d t),
$$


and losses

$$
\mathcal{L}_{B}=\int_{T_{B}^{-}}\left[\hat{P}_{t}^{A}\left(u_{t}\left(x_{A}^{*}\right)-u_{t}(y)\right)-\hat{P}_{t}^{A}\left(u_{t}\left(x_{A}^{*}\right)-u_{t}\left(x_{B}^{*}\right)\right)\right] \mu(d t) .
$$

By construction, the integrands of $\mathcal{G}_{B}$ and $\mathcal{L}_{B}$ are positive. Rewriting (7), we have $\mathcal{G}_{B} \leq \mathcal{L}_{B}$.

In the remainder of the proof, we show $\mathcal{L}_{A} \leq \mathcal{G}_{B}$ and $\mathcal{L}_{B} \leq \mathcal{G}_{A}$, at least one inequality strict. With (6) and (7), we then have

$$
\mathcal{G}_{A} \leq \mathcal{L}_{A} \leq \mathcal{G}_{B} \leq \mathcal{L}_{B} \leq \mathcal{G}_{A}
$$

at least one inequality strict, a contradiction. We first show $\mathcal{L}_{A} \leq \mathcal{G}_{B}$, a similar argument delivering $\mathcal{L}_{B} \leq \mathcal{G}_{A}$. To see that $T_{A}^{-} \subseteq T_{B}^{+}$, note that $t \in T_{A}^{-}$if and only if $u_{t}(y)<u_{t}\left(x_{A}^{*}\right)$; by concavity $u_{t}(y) \geq \frac{1}{2}\left[u_{t}\left(x_{A}^{*}\right)+u_{t}\left(x_{B}^{*}\right)\right]$; thus, $u_{t}(y)>u_{t}\left(x_{B}^{*}\right)$, or equivalently $t \in T_{B}^{+}$. Now take any $t \in T_{A}^{-}$and compare the integrands of $\mathcal{G}_{B}$ and $\mathcal{L}_{A}$ :

$$
\begin{aligned}
& \hat{P}_{t}^{A}\left(u_{t}\left(x_{A}^{*}\right)-u_{t}\left(x_{B}^{*}\right)\right)-\hat{P}_{t}^{A}\left(u_{t}\left(x_{A}^{*}\right)-u_{t}(y)\right) \\
& \quad \geq \hat{P}_{t}^{A}\left(u_{t}\left(x_{A}^{*}\right)-u_{t}\left(x_{B}^{*}\right)\right)-\hat{P}_{t}^{A}\left(u_{t}(y)-u_{t}\left(x_{B}^{*}\right)\right)
\end{aligned}
$$

if and only if

$$
\hat{P}_{t}^{A}\left(u_{t}\left(x_{A}^{*}\right)-u_{t}(y)\right) \leq \hat{P}_{t}^{A}\left(u_{t}(y)-u_{t}\left(x_{B}^{*}\right)\right) .
$$

Since $\hat{P}^{A}$ is strictly increasing, this holds if

$$
u_{t}(y)-u_{t}\left(x_{B}^{*}\right) \geq u_{t}\left(x_{A}^{*}\right)-u_{t}(y),
$$

or $u_{t}(y) \geq \frac{1}{2}\left[u_{t}\left(x_{A}^{*}\right)+u_{t}\left(x_{B}^{*}\right)\right]$, which indeed follows from concavity. This, together with the fact that the integrand in the definition of $\mathcal{G}_{B}$ is non-negative on $T_{B}^{+} \backslash T_{A}^{-}$, gives us $\mathcal{L}_{A} \leq \mathcal{G}_{B}$.

To prove that either $\mathcal{L}_{A}<\mathcal{G}_{B}$ or $\mathcal{L}_{B}<\mathcal{G}_{A}$, let $S=\left\{t \in T \mid u_{t}(y)>\frac{1}{2} u_{t}\left(x_{A}^{*}\right)+\frac{1}{2} u_{t}\left(x_{B}^{*}\right)\right\}$. By concavity, $S \subseteq T_{A}^{+} \cup T_{B}^{+}$. By aggregate strict concavity, $S$ has positive measure. Thus, without loss of generality, suppose $S \cap T_{B}^{+}$has positive measure. Since $T_{A}^{-} \subseteq T_{B}^{+}$, we then have either $\mu\left(S \cap T_{A}^{-}\right)>0$ or $\mu\left(S \cap\left(T_{B}^{+} \backslash T_{A}^{-}\right)\right)>0$. In the first case, for all $t \in S \cap T_{A}^{-}$, the inequality in (7) holds strictly and $\mathcal{L}_{A}<\mathcal{G}_{B}$ follows. In the second case, for all $t \in T_{B}^{+} \backslash T_{A}^{-}$, the integrand of $\mathcal{G}_{B}$ is positive, and the desired inequality follows.

\section{Robustness}

We next take up the issue of robustness of equilibria broached by Calvert (1985). To do so, we formulate candidate preferences more broadly. Namely, let $\Lambda$ be an arbitrary metric space of parameters with generic element $\lambda$, and let $U^{A}\left(x_{A}, x_{B}, \lambda\right)$ represent $A$ 's payoff. Similarly, let $U^{B}\left(x_{A}, x_{B}, \lambda\right)$ represent $B$ 's payoff. Let $E(\lambda)$ represent the set of mixed strategy equilibria of the electoral game corresponding to $\lambda$, where we give the space of mixed strategies the weak* topology. Let $\lambda^{*}$ be such that

$$
U^{C}\left(x_{A}, x_{B}, \lambda^{*}\right)=\int_{T} P_{t}^{C}\left(x_{A}, x_{B}\right) \mu(d t)
$$


$C \in\{A, B\}$. That is, $\lambda^{*}$ is the probabilistic voting model introduced in Section 2. Because $\lambda^{*}$ lies in a metric space, we can examine the robustness of equilibria when the parameters of our model are subject to small perturbations; that is, we can examine the continuity properties of $E$.

It may be, for example, that $\Lambda=[0,1]$, that candidates have preferences over platforms adopted given by continuous functions $u_{C}: X \rightarrow \Re$, that

$$
U^{C}\left(x_{A}, x_{B}, \lambda\right)=\lambda u_{C}\left(x_{C}\right)+(1-\lambda) \int_{T} P_{t}^{C}\left(x_{A}, x_{B}\right) \mu(d t),
$$

and hence that $\lambda^{*}=0$. Thus, we can examine the effects of small departures from our assumption of expected plurality share maximization. ${ }^{12}$

Theorem 9 Assume (i) $X$ is compact and (ii) for each $C, U^{C}\left(x_{A}, x_{B}, \lambda\right)$ is jointly continuous in $\left(x_{A}, x_{B}, \lambda\right)$. Then $E$ has closed graph.

Proof: The result follows from upper hemicontinuity of the mixed strategy equilibrium correspondence, which is well-known.

Because $\Lambda$ is an arbitrary metric space, $\lambda$ may index distributions of voter types, and we can consider robustness with respect to changes in demographic variables. Or we can let $\lambda$ index families of expected plurality share functions, and we can examine the effects of small changes in the underlying structure of incomplete information in the electoral game. We first apply Theorem 9 to establish robustness of equilibria of the electoral game with respect to demographic variables captured by the distribution of types, $\mu$.

Corollary 5 Assume (i) $X$ is compact, (ii) $T$ is a compact metric space, (iii) for each $C, P_{t}^{C}\left(x_{A}, x_{B}\right)$ is jointly continuous in $\left(t, x_{A}, x_{B}\right)$, (iv) $\mu^{m} \rightarrow \mu$ weakly, and (iv) for each $m,\left(\pi_{A}^{m}, \pi_{B}^{m}\right)$ is a mixed strategy equilibrium of the electoral game parameterized by $\mu^{m}$. If $\left(\pi_{A}^{m}, \pi_{B}^{m}\right) \rightarrow\left(\pi_{A}, \pi_{B}\right)$, then $\left(\pi_{A}, \pi_{B}\right)$ is a mixed strategy equilibrium of the electoral game parameterized by $\mu$.

Proof: The result follows from Theorem 9, where $\Lambda$ is the space of probability measures on $(T, \mathcal{T})$ endowed with the weak* topology. Define

$$
U^{C}\left(x_{A}, x_{B}, \mu\right)=\int_{T} P_{t}^{C}\left(x_{A}, x_{B}\right) \mu(d t),
$$

$C \in\{A, B\}$. To establish continuity of $U^{C}$, let $\left\{\left(x_{A}^{m}, x_{B}^{m}, \mu^{m}\right)\right\}$ converge to $\left(x_{A}, x_{B}, \mu\right)$. Given $\lambda$ and $x_{A}$, define $\phi: \Lambda \rightarrow \Re$ by

$$
\psi(y, z)=\max _{t \in T}\left|P_{t}^{C}(y, z)-P_{t}^{C}\left(x_{A}, x_{B}\right)\right| .
$$

\footnotetext{
${ }^{12}$ In this example, candidates have preferences over their respective platforms. Because we have formulated the candidates' objective functions in terms of expected plurality share, rather than probability of winning, we have no way of naturally introducing preferences over policies implemented by the winner, as Calvert does. Because he assumes probability of winning functions are continuous, his Theorem 6 follows from known continuity results, as Theorem 9 does.
} 
This function is continuous by the Theorem of the Maximum, and $\phi\left(x_{A}, x_{B}\right)=0$. Therefore $\phi\left(x_{A}^{m}, x_{B}^{m}\right) \rightarrow 0$. That is, $P_{(\cdot)}^{C}\left(x_{A}^{m}, x_{B}^{m}\right)-P_{(\cdot)}^{C}\left(x_{A}, x_{B}\right) \rightarrow 0$ uniformly, and

$$
\int_{T} P_{t}^{C}\left(x_{A}, x_{B}\right) \mu^{m}(d t) \rightarrow \int_{T} P_{t}^{C}\left(x_{A}, x_{B}\right) \mu(d t),
$$

follows from Aliprantis and Border's (1994) Corollary 12.6.

We now apply Theorem 9 to establish that equilibria of the electoral game are robust with respect to variations in the underlying structure of incomplete information in the model: given one specification of expected plurality share functions, "nearby" functions will not admit equilibria far away from those of the first. Much hinges on the meaning of "nearby" in reference to expected plurality share functions. In our next theorem, we use the notion of uniform convergence, and hence "nearby" has a very strong meaning.

Corollary 6 Assume (i) $X$ is compact, (ii) for each t and $C,\left\{P_{t}^{C, m}\right\}$ is a sequence of continuous functions converging uniformly to $P_{t}^{C}$ and (iii) for each $m,\left(\pi_{A}^{m}, \pi_{B}^{m}\right)$ is a mixed strategy equilibrium of the electoral game parameterized by $\left\{P_{t}^{C, m}\right\}_{t, C} . \quad$ If $\left(\pi_{A}^{m}, \pi_{B}^{m}\right) \rightarrow$ $\left(\pi_{A}, \pi_{B}\right)$, then $\left(\pi_{A}, \pi_{B}\right)$ is a mixed strategy equilibrium of the game parameterized by $\left\{P_{t}^{C}\right\}_{t, C}$.

Proof: The result follows from Theorem 9, where $\Lambda=\left(\Pi_{t} \mathcal{P}\right) \times\left(\Pi_{t} \mathcal{P}\right)$ and $\mathcal{P}$ is the space of continuous expected plurality share functions. We endow $\mathcal{P}$ with the topology of uniform convergence and $\Lambda$ with the product topology. Define

$$
U^{C}\left(x_{A}, x_{B},\left\{P_{t}^{C}\right\}_{t, C}\right)=\int_{T} P_{t}^{C}\left(x_{A}, x_{B}\right) \mu(d t),
$$

$C \in\{A, B\}$. To establish continuity of $U^{C}$, let $\left\{\left(x_{A}^{m}, x_{B}^{m},\left\{P_{t}^{C, m}\right\}_{t, C}\right)\right\}$ converge to $\left(x_{A}, x_{B}\right.$, $\left.\left\{P_{t}^{C}\right\}_{t, C}\right)$. Take any $t$, and note that, because $P_{t}^{C}$ is continuous and $P_{t}^{C, m} \rightarrow P_{t}^{C}$ uniformly, $P_{t}^{C, m}\left(x_{A}^{m}, x_{B}^{m}\right) \rightarrow P_{t}^{C}\left(x_{A}, x_{B}\right)$. Therefore,

$$
U^{C}\left(x_{A}^{m}, x_{B}^{m},\left\{P_{t}^{C, m}\right\}_{t, C}\right) \rightarrow U^{C}\left(x_{A}, x_{B},\left\{P_{t}^{C}\right\}_{t, C}\right)
$$

follows from Lebesgue's dominated convergence theorem.

We can weaken uniform convergence to pointwise convergence if we go to the Utility Difference Model and make the weak assumption that expected plurality share functions of the candidates are weakly monotonic. This gives us a much weaker notion of "nearby" and a stronger robustness result.

Corollary 7 In the Utility Difference Model, assume (i) $X$ is compact, (ii) for each $t$, $u_{t}$ is continuous, (iii) for each $t$ and $C, \hat{P}_{t}^{C}$ is continuous, (iv) for each $t$ and $C,\left\{\hat{P}_{t}^{C, m}\right\}$ is a sequence of weakly monotonic functions converging pointwise to $\hat{P}_{t}^{C}$, and $(v)$ for each $m$, $\left(\pi_{A}^{m}, \pi_{B}^{m}\right)$ is a mixed strategy equilibrium of the electoral game parameterized by $\left\{\hat{P}^{C, m}\right\}_{t, C}$. If $\left(\pi_{A}^{m}, \pi_{B}^{m}\right) \rightarrow\left(\pi_{A}, \pi_{B}\right)$, then $\left(\pi_{A}, \pi_{B}\right)$ is a mixed strategy equilibrium of the game parameterized by $\left\{\hat{P}_{t}^{C}\right\}_{t, C}$. 
Proof: The result follows from Theorem 9, where $\Lambda=\left\{\left\{\hat{P}_{t}^{C, m}\right\}_{t, C} \mid m=1,2, \ldots\right\} \cup$ $\left\{\left\{\hat{P}_{t}^{C}\right\}_{t, C}\right\}$ is endowed with a simple topology: only $\left\{\left\{\hat{P}_{t}^{C, m}\right\}_{t, C}\right\}$ and its subseqences converge. Define

$$
U^{C}\left(x_{A}, x_{B},\left\{\hat{P}_{t}^{C}\right\}_{t, C}\right)=\int_{T} \hat{P}_{t}^{C}\left(u_{t}\left(x_{A}\right)-u_{t}\left(x_{B}\right)\right) \mu(d t)
$$

$C \in\{A, B\}$. To establish continuity of $U^{C}$, let $\left\{\left(x_{A}^{m}, x_{B}^{m},\left\{\hat{P}_{t}^{C, m}\right\}_{t, C}\right)\right\}$ converge to $\left(x_{A}, x_{B}\right.$, $\left.\left\{\hat{P}_{t}^{C}\right\}_{t, C}\right)$. Take any $t$ and $C$, and note that $\left\{\hat{P}_{t}^{C, m}\right\}$ is a sequence of weakly monotonic functions converging pointwise to $\hat{P}_{t}^{C}$ on the interval $\left[u_{t}\left(x_{A}\right)-u_{t}\left(x_{B}\right)-1, u_{t}\left(x_{A}\right)-u_{t}\left(x_{B}\right)+1\right]$. Therefore, $\left\{\hat{P}_{t}^{C, m}\right\}$ converges uniformly on this interval. (See Aliprantis and Burkinshaw, 1990, Sec.7, ex.14.) The proof then proceeds as in the proof of Corollary 6.

\section{Core Convergence}

In the context of the Utility Difference Model, we now take up a different kind of robustness issue. Define the Deterministic Model by the expected plurality share functions $\bar{P}_{t}^{A}$ and $\bar{P}_{t}^{B}$, where

$$
\bar{P}_{t}^{A}(u)= \begin{cases}1 & \text { if } u>0 \\ 0 & \text { if } u=0 \\ -1 & \text { if } u<0\end{cases}
$$

and $\bar{P}_{t}^{B}=-\bar{P}_{t}^{A}$. We parameterize voter preferences by $\lambda$, as in $u_{t}(x, \lambda)$, where $\lambda$ again lies in an arbitrary metric space. We define the core at $\lambda$ as the set of policies $x \in X$ for which there does not exist $y \in X$ such that

$$
\mu\left(\left\{t \in T \mid u_{t}(y, \lambda)>u_{t}(x, \lambda)\right\}\right)>\frac{1}{2} .
$$

It is clear that the pure strategy equilibrium platforms of the electoral game, when the Deterministic Model obtains, must lie within the core. Thus, if the core is empty, then pure strategy equilibria will not exist. Suppose that the core at $\lambda$ is strongly externally stable, i.e., for every $x$ in the core at $\lambda$ and for every other $y$, we have $\mu\left(\left\{t \in T \mid u_{t}(x, \lambda)>\right.\right.$ $\left.\left.u_{t}(y, \lambda)\right\}\right)>\frac{1}{2}$. If the core is non-empty and strongly externally stable, then we can say more: the core consists of one point, $x^{*}$, and $\left(x_{A}, x_{B}\right)$ is a pure strategy equilibrium if and only if $x_{A}=x_{B}=x^{*}$. Further, the equilibrium mixed strategies are then exactly those placing probability one on $x^{*}$.

It is well known that core points must meet stringent conditions and that, even when the core is non-empty, small perturbations of voter preferences may annihilate it (see Plott (1967)). In this section, we show that small perturbations of preferences and small departures from the Deterministic Model cannot produce electoral equilibria "far away" from the core, if non-empty. More than that, if pure strategy equilibria exist for models arbitrarily close to the Deterministic Model, then the core must be non-empty. Because of discontinuities inherent in the Deterministic Model, a difficult technical issue not addressed in 
the previous section, Theorem 9 and Corollary 7 do not yield the desired core convergence results, so that separate arguments are required.

Our first theorem on core convergence establishes a robustness result using minimal background assumptions: models nearby the Deterministic Model cannot possess pure strategy electoral equilibria far away from the core. We then extend this result to mixed strategies by assuming that the core at a parametrization $\lambda$ is weakly externally stable, i.e., for every $x$ in the core at $\lambda$ and for every other $y$, we have $\mu\left(\left\{t \in T \mid u_{t}(x, \lambda)>u_{t}(y, \lambda)\right\}\right) \geq \frac{1}{2}$, replacing the strict inequality above with a weak one. This holds, for example, if $X$ is convex and each $u_{t}$ is strictly quasi-concave. We find that, if there exist mixed strategy electoral equilibria of models close to deterministic that converge to a pure strategy pair, then those pure strategy platforms must be in the core. In the following, let $\delta_{x}$ denote the probability measure with point mass on $x$.

Theorem 10 In the Utility Difference Model, assume (i) for each $t, u_{t}(x, \lambda)$ is jointly continuous in $(x, \lambda)$, (ii) $\left\{\lambda^{m}\right\}$ is a sequence converging to $\lambda$, and (iii) for each $t$ and $C$, $\left\{\hat{P}_{t}^{C, m}\right\}$ is a sequence of weakly monotonic functions converging pointwise to $\bar{P}_{t}^{C}$. For each $m$, let $\left(x_{A}^{m}, x_{B}^{m}\right)$ be a pure strategy equilibrium of the electoral game parameterized by $\lambda^{m}$ and $\left\{\hat{P}_{t}^{C, m}\right\}_{t, C}$. If $\left(x_{A}^{m}, x_{B}^{m}\right) \rightarrow\left(x_{A}, x_{B}\right)$, then $x_{A}$ and $x_{B}$ are in the core at $\lambda$.

Assume, moreover, (iv) the core at $\lambda$ is weakly externally stable. For each $m$, let $\left(\pi_{A}^{m}, \pi_{B}^{m}\right)$ be a mixed strategy equilibrium of the electoral game parameterized by $\lambda^{m}$ and $\left\{\hat{P}_{t}^{C, m}\right\}_{t, C}$. If $\left(\pi_{A}^{m}, \pi_{B}^{m}\right) \rightarrow\left(\delta_{x_{A}}, \delta_{x_{B}}\right)$, then $x_{A}$ and $x_{B}$ are in the core at $\lambda$.

Proof: For the first part of the theorem, suppose $x_{A}$ is not in the core at $\lambda$, so that $\mu(\{t \in$ $\left.\left.T \mid u_{t}(y, \lambda)>u_{t}\left(x_{A}, \lambda\right)\right\}\right)>\frac{1}{2}$ for some $y \in X$. Let $S=\left\{t \in T \mid u_{t}(y, \lambda)>u_{t}\left(x_{A}, \lambda\right)\right\}$, so that

$$
\int_{S} \bar{P}_{t}^{B}\left(u_{t}\left(x_{A}, \lambda\right)-u_{t}(y, \lambda)\right) \mu(d t)=\mu(S),
$$

and let $a<1$ satisfy $a>2 \mu(T \backslash S)$. Take any $t \in S$, and note that from (i), (ii), and $x_{A}^{m} \rightarrow x_{A}$, there exists $\epsilon>0$ and $M$ such that, for $m \geq M, u_{t}\left(y, \lambda^{m}\right)-u_{t}\left(x_{A}^{m}, \lambda^{m}\right)>\epsilon$. By (iii), we have $\hat{P}_{t}^{B, m}(-\epsilon)>a$ for high enough $m$. And since $\hat{P}_{t}^{B, m}$ is weakly decreasing, $\hat{P}_{t}^{B, m}\left(u_{t}\left(x_{A}^{m}, \lambda^{m}\right)-u_{t}\left(y, \lambda^{m}\right)\right)>a$ for high enough $m$. By Fatuou's Lemma,

$$
\begin{aligned}
\int_{S} \hat{P}_{t}^{B, m}\left(u_{t}\left(x_{A}^{m}, \lambda^{m}\right)-u_{t}\left(y, \lambda^{m}\right)\right) \mu(d t) & \geq a \mu(S) \\
\int_{T \backslash S} \hat{P}_{t}^{B, m}\left(u_{t}\left(x_{A}^{m}, \lambda^{m}\right)-u_{t}\left(y, \lambda^{m}\right)\right) \mu(d t) & \geq-(2-a) \mu(T \backslash S),
\end{aligned}
$$

and therefore,

$$
\int_{T} \hat{P}_{t}^{B, m}\left(u_{t}\left(x_{A}^{m}, \lambda^{m}\right)-u_{t}\left(y, \lambda^{m}\right)\right) \mu(d t) \geq a-2 \mu(T \backslash S)
$$

for high enough $m$. For such $m$, since $x_{B}^{m}$ is a best response to $x_{A}^{m}$, it must be that

$$
\int_{T} \hat{P}_{t}^{B, m}\left(u_{t}\left(x_{A}^{m}, \lambda^{m}\right)-u_{t}\left(x_{B}^{m}, \lambda^{m}\right)\right) \mu(d t) \geq a-2 \mu(T \backslash S)>0 .
$$


It follows that candidate $A$ 's payoff is less than $\mu(T \backslash S)-\frac{a}{2}<0$ for high enough $m$. Since $\hat{P}_{t}^{A, m}(0) \rightarrow \bar{P}_{t}^{A}(0)=0$ for each $t$, Lebesgue's dominated convergence theorem yields

$$
\int_{T} \hat{P}_{t}^{A, m}(0) \mu(d t)>\mu(T \backslash S)-\frac{a}{2}
$$

for high enough $m$. But $x_{B}^{m}$ is then a better response to $x_{B}^{m}$ for $A$ than $x_{A}^{m}$, and $\left(x_{A}^{m}, x_{B}^{m}\right)$ cannot be an electoral equilibrium. A symmetric argument addresses the case in which $x_{B}$ is not in the core.

For the second part, suppose $x_{A}$ is not in the core at $\lambda$, so that again $\mu(\{t \in T \mid$ $\left.\left.u_{t}(y, \lambda)>u_{t}\left(x_{A}, \lambda\right)\right\}\right)>\frac{1}{2}$ for some $y \in X$. By Proposition 13 in Banks, Duggan, and Le Breton (2003), there exist a measurable set $S \subseteq T$ with $\mu(S)>\frac{1}{2}$ and an open set $Y \subseteq \Re^{n}$ such that $y \in Y$ and, for all $t \in S$ and all $z \in X \cap Y, u_{t}(y, \lambda)>u_{t}(z, \lambda)$. Let $a<1$ satisfy $a>2 \mu(T \backslash S)$. From $\pi_{A}^{m} \rightarrow \delta_{x_{A}}$, we have

$$
\pi_{A}^{m}(X \backslash Y)<[a-2 \mu(T \backslash S)] \pi_{A}^{m}(Y),
$$

for high enough $m$. Take any any $z \in Y$ and any $t \in S$, and note that, by (i) and (ii), there exists $\epsilon>0$ and $M$ such that, for $m \geq M, u_{t}\left(y, \lambda^{m}\right)-u_{t}\left(z, \lambda^{m}\right)>\epsilon$. By (iii), we have $\hat{P}_{t}^{B, m}(-\epsilon)>a$ for high enough $m$. And since $\hat{P}_{t}^{B, m}$ is weakly decreasing, we have $\hat{P}_{t}^{B, m}\left(u_{t}\left(x_{A}^{m}, \lambda^{m}\right)-u_{t}\left(y, \lambda^{m}\right)\right)>a$ for high enough $m$. For any $z \in Y$, we therefore again have (8)-(10), with $x_{A}^{m}$ replaced by $z$. Therefore,

$$
\begin{aligned}
\int_{X} & \int_{T} \hat{P}_{t}^{B, m}\left(u_{t}\left(z, \lambda^{m}\right)-u_{t}\left(y, \lambda^{m}\right)\right) \mu(d t) \pi_{A}^{m}(d z) \\
= & \int_{Y} \int_{T} \hat{P}_{t}^{B, m}\left(u_{t}\left(z, \lambda^{m}\right)-u_{t}\left(y, \lambda^{m}\right)\right) \mu(d t) \pi_{A}^{m}(d z) \\
& \quad+\int_{X \backslash Y} \int_{T} \hat{P}_{t}^{B, m}\left(u_{t}\left(z, \lambda^{m}\right)-u_{t}\left(y, \lambda^{m}\right)\right) \mu(d t) \pi_{A}^{m}(d z) \\
& \geq[a-2 \mu(T \backslash S)] \pi_{A}^{m}(Y)-\pi_{A}^{m}(X \backslash Y) \\
> & 0
\end{aligned}
$$

for high enough $m$. For such $m$, since $\pi_{B}^{m}$ is a best response to $\pi_{A}^{m}$, it must be that candidate $B$ 's expected payoff from using $\pi_{B}^{m}$ against $\pi_{A}^{m}$ is at least $a-2 \mu(T \backslash S)-\pi_{A}^{m}(X \backslash Y)>0$. This implies that candidate $A$ 's expected payoff from using $\pi_{A}^{m}$ against $\pi_{B}^{m}$ is less than $-\frac{a}{2}+\mu(T \backslash S)+\frac{\pi_{A}^{m}(X \backslash Y)}{2}<0$ for high enough $m$. To derive a contradiction, note that either $x_{B}$ is in the core at $\lambda$ or not. In the latter case, there is some $y \in X$ such that $\mu\left(\left\{t \in T \mid u_{t}(y, \lambda)>u_{t}\left(x_{B}, \lambda\right)\right\}\right)>\frac{1}{2}$, and the above argument shows that $A$ 's expected payoff must be positive for high enough $m$, a contradiction. In the former case, let $y=x_{B}$. Using (iv), we can argue as above that

$$
\liminf \int_{X} \int_{T} \hat{P}_{t}^{A, m}\left(u_{t}\left(y, \lambda^{m}\right)-u_{t}\left(x_{B}, \lambda^{m}\right)\right) \mu(d t) \pi_{B}^{m}\left(d x_{B}\right) \geq 0,
$$

again a contradiction. A symmetric argument addresses the case in which $x_{B}$ is not in the core. 
Note that condition (iv) in the second part of Theorem 10 could be replaced by the symmetry condition in Theorem 7 , which reduces to $\hat{P}_{t}^{A}(u)=\hat{P}_{t}^{B}(-u)$ in the Utility Difference Model. Condition (iv) is used in the proof only to argue that candidate $A$ could guarantee a (close to) non-negative expected payoff against $\pi_{B}^{m}$ for high enough $m$. In the proof, this was accomplished by letting $A$ choose a platform in the core, and under the symmetry condition this could be done by letting $A$ adopt $B$ 's mixed strategy.

Note that Theorem 10 does not require the assumption that the core at $\lambda$ is nonempty. As a consquence, when the core is empty, the implications of Theorem 10 are definitive: pure strategy equilibria are guaranteed to not exist in models close enough to deterministic. Though Theorem 2 gives conditions under which pure strategy equilibria would exist, they cannot exist in or "near" the Deterministic Model when the core is empty. The problem is that, because of non-concavities inherent in the Deterministic Model, the concavity condition needed in Theorem 2 is necessarily violated in models close enough to the Deterministic Model. Of course, there is no guarantee that pure strategy will exist after perturbing the Deterministic Model, even when the core is non-empty. We return to a related issue in the next section.

Corollary 8 In the Utility Difference Model, assume (i) $X$ is compact, (ii) for each $t$, $u_{t}(x, \lambda)$ is jointly continuous in $(x, \lambda)$, (iii) $\left\{\lambda^{m}\right\}$ is a sequence converging to $\lambda$, and (iv) for each $t$ and $C,\left\{\hat{P}_{t}^{C, m}\right\}$ is a sequence of weakly monotonic functions converging pointwise to $\bar{P}_{t}^{C}$. If the core at $\lambda$ is empty, then there exists $M$ such that, for all $m \geq M$, the electoral game parameterized by $\lambda^{m}$ and $\left\{\hat{P}_{t}^{C, m}\right\}_{t, C}$ has no pure strategy electoral equilibria.

Proof: If the conclusion of the corollary did not hold, we could extract a subsequence $\left\{\left(x_{A}^{m_{k}}, x_{B}^{m_{k}}\right)\right\}$ of pure strategy equilibria converging to some $\left(x_{A}, x_{B}\right)$. By the first part of Theorem $10, x_{A}$ and $x_{B}$ would be in the core at $\lambda$, a contradiction.

Finally, we extend the second part of Theorem 10 by addressing the possibility of mixed strategy electoral equilibria in models that do not converge to pure strategies. We assume that the core at $\lambda$ is non-empty and strongly externally stable, the latter holding if $X$ is convex, each $u_{t}$ is strictly quasi-concave, and majority rule is strong. ${ }^{13}$ We find that in models close to the Deterministic Model, mixed strategy electoral equilibria must be close to the unique core point.

Theorem 11 In the Utility Difference Model, assume (i) for each $t, u_{t}(x, \lambda)$ is jointly continuous in $(x, \lambda)$, (ii) $\left\{\lambda^{m}\right\}$ is a sequence converging to $\lambda$, (iii) for each $t$ and $C,\left\{\hat{P}_{t}^{C, m}\right\}$ is a sequence of weakly monotonic functions converging pointwise to $\bar{P}_{t}^{C}$, and (iv) the core at $\lambda$ is strongly externally stable and consists of the policy $x^{*}$. For each $m$, let $\left(\pi_{A}^{m}, \pi_{B}^{m}\right)$ be a mixed strategy equilibrium of the electoral game parameterized by $\lambda^{m}$ and $\left\{\hat{P}_{t}^{C, m}\right\}_{t, C}$. Then $\left(\pi_{A}^{m}, \pi_{B}^{m}\right) \rightarrow\left(\delta_{x^{*}}, \delta_{x^{*}}\right)$.

\footnotetext{
${ }^{13}$ Banks, Duggan, and Le Breton (2003) show that majority rule need not be strong for this claim when $T$ is a continuum if voter preferences are sufficiently heterogeneous.
} 
Proof: We claim that $\phi: X \rightarrow \Re$ defined by

$$
\phi(z)=\mu\left(\left\{t \in T \mid u_{t}\left(x^{*}, \lambda\right)>u_{t}(z, \lambda)\right\}\right)
$$

is lower semicontinuous. Let $S_{z}=\left\{t \in T \mid u_{t}\left(x^{*}, \lambda\right)>u_{t}(z, \lambda)\right\}$. Given a sequence $z^{m} \rightarrow z$, by (ii) we have $S_{z} \subseteq \liminf S_{z^{m}}$. Then the claim follows from

$$
\liminf \mu\left(S_{z^{m}}\right) \geq \mu\left(\liminf S_{z^{m}}\right) \geq \mu\left(S_{z}\right),
$$

an implication of Fatou's Lemma. Now suppose $\left\{\pi_{A}^{m}\right\}$ does not converge weakly to $\delta_{x^{*}}$. Then there is some $b>0$ and some open set $Y$ containing $x^{*}$ such that, for all $M$, there exists $m \geq M$ with $\pi_{A}^{m}(X \backslash Y) \geq b$. Going to a subsequence if needed, we can suppose this is true of all $m$. From (i) and lower semicontinuity of $\phi, \phi$ achieves it's minimum, say $\nu$, on $X \backslash Y$. From (iv), we have $\nu>\frac{1}{2}$. Let $a<1$ satisfy $a>2(1-\nu)$. Take any $z \in X \backslash Y$ and any $t \in S_{z}$. By (ii) and (iii), there exists $\epsilon_{z}>0$ and $M_{z}$ such that, for $m \geq M_{z}$, we have $u_{t}\left(x^{*}, \lambda^{m}\right)-u_{t}\left(z, \lambda^{m}\right)>\epsilon_{z}$. By (iii), we have $\hat{P}_{t}^{B, m}\left(-\epsilon_{z}\right)>a$ for high enough $m$. And then since $\hat{P}_{t}^{B, m}$ is weakly decreasing, $\hat{P}_{t}^{B, m}\left(u_{t}\left(z, \lambda^{m}\right)-u_{t}\left(x^{*}, \lambda^{m}\right)\right)>a$ for high enough $m$. For any $z \in X \backslash Y$, Fatou's Lemma implies

$$
\begin{aligned}
\int_{T} \hat{P}_{t}^{B, m}\left(u_{t}\left(z, \lambda^{m}\right)-u_{t}\left(x^{*}, \lambda^{m}\right)\right) \mu(d t) & \geq a \mu\left(S_{z}\right)-\mu\left(T \backslash S_{z}\right) \\
& \geq a-2(1-\nu)
\end{aligned}
$$

for high enough $m$. Therefore,

$$
\int_{X \backslash Y} \int_{T} P_{t}^{B, m}\left(u_{t}\left(z, \lambda^{m}\right)-u_{t}\left(x^{*}, \lambda^{m}\right)\right) \mu(d t) \pi_{A}^{m}(d z) \geq(a-2(1-\nu)) b>0,
$$

for high enough $m$. Taking $m$ high enough, a similar argument shows that

$$
\int_{Y} \int_{T} P_{t}^{B, m}\left(u_{t}\left(z, \lambda^{m}\right)-u_{t}\left(x^{*}, \lambda^{m}\right)\right) \mu(d t) \pi_{A}^{m}(d z)
$$

can be bounded below by negative numbers arbitrarily close to zero. Therefore, there exists $c>0$ such that $B$ 's expected payoff using $x^{*}$ against $\pi_{A}^{m}$ is at least $c$ for high enough $m$. Since $\pi_{B}^{m}$ is a best response to $\pi_{A}^{m}, B$ 's expected payoff from using $\pi_{B}^{m}$ against $\pi_{A}^{m}$ is at least $c$ for high enough $m$, and therefore $A$ 's expected payoff is less than or equal to $-c$. Finally, an argument similar to that above yields a lower bound on $A$ 's expected payoff using $x^{*}$ against $\pi_{B}^{m}$ that goes to zero. But then, for high enough $m, x^{*}$ is a better response to $\pi_{B}^{m}$ for $A$ than $\pi_{A}^{m}$, a contradiction. A symmetric argument addresses the case in which $\left\{\pi_{B}^{m}\right\}$ does not converge to $\delta_{x^{*}}$.

It is important to note a difference between Theorem 10 and Theorem 11: non-emptiness of the core at $\lambda$ is a conclusion in the latter, allowing us to derive Corollary 8 , whereas in the former it is an assumption. If we drop the assumption of non-emptiness and have a convergent sequence of mixed strategy equilibria, can we deduce non-emptiness of the core? Evidently not: even when the core is empty, the Deterministic Model can be approximated by continuous models generating a convergent sequence of mixed strategy equilibria. From the second part of Theorem 10, however, we can say that such a sequence of mixed strategy electoral equilibria in nearby models could not converge to a pure strategy electoral equilibrium in the Deterministic Model. 


\section{Is the Median an Artifact?}

In two papers, Hinich $(1977,1978)$ has suggested that the median voter result of Black (1958) and Downs (1957) is an "artifact" of their assumption of deterministic voting behavior. In the earlier of his papers, Hinich assumes three voters, quadratic utility functions with median ideal point distinct from the mean, and he approximates the one-dimensional Deterministic Model with a sequence of continuous expected plurality share functions converging pointwise to the Deterministic Model. He finds that they do not possess pure strategy equilibria close to the median and that, if the continuous models do have pure strategy equilibria, then they must be at the mean rather than the median. Hinich gives an example, 'Convergence to the Mean,' suggesting that, arbitrarily close to the Deterministic Model, the mean is indeed an equilibrium. Thus, the median appears fragile: adding the slightest uncertainty, the equilibrium jumps to the mean.

In the later of the papers, Hinich generalizes his earlier observations, allowing for a continuum of voters, multiple policy dimensions, and generalized Euclidean preferences. His Theorem 1 (see our Corollary 4) establishes that, in the presence of uncertainty about voters' choices, if pure strategy equilibria of the electoral game exist, then the candidates must locate at the mean ideal point. His Theorem 2, proved in the context of the Additive Bias Model with normally distributed bias and a one-dimensional policy space, states that, for small enough variance, the mean is a pure strategy equilibrium of the electoral game. If this were true, and if the mean were distinct from the median, then the equilibrium would jump from the median to the mean in the presence of the slightest uncertainty. Hinich does not consider mixed strategies.

Our Theorems 5 and 10 can be used to formalize and clarify the suggested fragility of the median. Suppose, for example, that the median and mean are distinct, and that voters have quadratic utility functions. Consider a sequence of normally distributed Additive Bias Models with variance converging to zero (generating a sequence of continuous expected plurality share functions converging pointwise to the Deterministic Model), and suppose that, indeed, pure strategy equilibria exist for small enough variance. By Corollary 4, we know that the equilibrium platforms of the candidates are at the mean as the variance gets close to zero. By the first part of Theorem 10, however, this sequence of equilibria must converge to the core, i.e., the median. But the median is distinct from the mean, an impossibility. Given Corollary 4 and Theorem 10, pure strategy equilibria must fail to exist beyond some point in this sequence, i.e., there is some variance level such that any Additive Bias Models with smaller variance fail to admit pure strategy equilibria, contradicting Hinich's (1978) Theorem 2. Thus, though the median must cease to be an equilibrium when arbitrarily small amounts of uncertainty are added to the Deterministic Model, the equilibrium cannot jump to the mean.

A further observation arises from Theorems 1 and 10. Namely, we know from Theorem 1 that mixed strategy equilibria do exist as the variance of the bias term goes to zero. And we know from the third part of Theorem 10 that these equilibrium mixed strategies must converge weak* to the median. In other words, given an arbitrarily small open interval around the median, the probability that the equilibrium mixed strategies of the candidates determine policies within that open interval goes to one. Thus, we may expect outcomes 
close to the median, even when small amounts of uncertainty are added to the Deterministic Model.

The next theorem restates these observations formally, echoing Kramer's (1978) criticism of Hinich's earlier analysis. Kramer also argues that Hinich's examples fail to illustrate the fragility of the median, and, assuming candidates are motivated by probability of winning, he proves that mixed strategy equilibria of models close to the Deterministic Model must converge to the median. ${ }^{14}$ We offer similar conclusions in the expected plurality maximizing framework of Hinich, and in the more general context of a multidimensional policy space and general concave voter utility functions. To facilitate further discussion of Hinich's examples, we also allow the voters' preferences to vary.

Theorem 12 In the Utility Difference Model, assume (i) $X$ is compact and convex, (ii) for each $t, u_{t}(x, \lambda)$ is continuous in $(x, \lambda)$, (iii) $\left\{\lambda^{m}\right\}$ is a sequence converging to $\lambda$, (iv) for each $t$ and $m, u_{t}\left(x, \lambda^{m}\right)$ is concave in $x$, ( $v$ ) for each $m$, aggregate strict concavity holds, (vi) for each $t$ and $C,\left\{\hat{P}_{t}^{C, m}\right\}$ is a sequence of strictly monotonic functions converging pointwise to $\bar{P}_{t}^{C}$, and (vii) for each $t, C$, and $m, \hat{P}_{t}^{C, m}(u)$ is differentiable with derivatives bounded in $(t, u)$ and with $D_{u=0} \hat{P}_{t}^{C, m}(u)$ independent of $t$. If the core at $\lambda$ is disjoint from

$$
\arg \max _{x} \int u_{t}(x, \lambda) \mu(d t),
$$

then there exists $M$ such that, for all $m \geq M$, the electoral game parameterized by $\lambda^{m}$ and $\left\{\hat{P}_{t}^{C, m}\right\}_{t, C}$ has no pure strategy electoral equilibria.

Assume, moreover, (viii) the core is strongly externally stable and consists of just one policy $x^{*}$. For each $m$, there exists a mixed strategy equilibrium $\left(\pi_{A}^{m}, \pi_{B}^{m}\right)$ of the electoral game parameterized by $\lambda^{m}$ and $\left\{\hat{P}_{t}^{C, m}\right\}_{t, C}$. For all sequences of such mixed strategy equilibria, $\left(\pi_{A}^{m}, \pi_{B}^{m}\right) \rightarrow\left(\delta_{x^{*}}, \delta_{x^{*}}\right)$.

Proof: Suppose the first conclusion of the theorem is false. Then we can extract a subsequence $\left\{\left(x_{A}^{m_{k}}, x_{B}^{m_{k}}\right)\right\}$ of pure strategy equilibria. By Theorem $8, x_{A}^{m_{k}}=x_{B}^{m_{k}}$ for all $k$. Then, by Corollary 3, we have

$$
x_{A}^{m_{k}}=x_{B}^{m_{k}} \in \arg \max _{x} \int u_{t}\left(x, \lambda^{m_{k}}\right) \mu(d t)
$$

for all $k$. By (i), $\left\{\left(x_{A}^{m_{k}}, x_{B}^{m_{k}}\right)\right\}$ has a subsequence converging to some $\left(x_{A}, x_{B}\right)$. By (i), (ii), and the Theorem of the Maximum,

$$
x_{A}=x_{B} \in \arg \max _{x} \int u_{t}(x, \lambda) \mu(d t) .
$$

But by the first part of Theorem 10, $x_{A}$ and $x_{B}$ are in the core at $\lambda$, a contradiction. By the third part of Theorem 1, mixed strategy equilibria exist for each $m$. Since $X$ is compact, so is the space of mixed strategy pairs, and so every sequence of mixed strategy pairs has a limit point. Under (viii), the third part of Theorem 10 yields $x^{*}$ as the only limit point.

\footnotetext{
${ }^{14}$ In fact, he proves something stronger: each non-median platform is strictly dominated in models close enough to the Deterministic Model.
} 
There are two points where Theorem 12 must be reconciled with Hinich's work. The first point is Hinich's (1977) 'Convergence to the Mean' example. Here, we have voters with quadratic utility functions and a sequence of continuous expected plurality share functions, parameterized by $\delta$, converging to the Deterministic Model. But given a particular $\delta$, the candidates' objective functions are concave only within an interval of the mean, creating a problem for existence of pure strategy equilibria. This concavity problem forces Hinich to arrange voter ideal points within this interval, which itself depends on $\delta$ and collapses to the mean as $\delta$ goes to zero. Thus, we cannot interpret this example as adding an arbitrarily small amount of uncertainty to the Deterministic Model. Furthermore, Hinich's argument does not simply require "small" movements of ideal points, for the voters' ideal points will have to be collapsed to within an arbitrarily small neighborhood of the mean as $\delta$ goes to zero. Theorem 12, because it allows the voters' utility functions to vary with the expected plurality share functions, formalizes the point that, as expected plurality share functions converge to the Deterministic Model, the mean cannot be made an equilibrium by arbitrarily small changes in voter preferences.

The second point is Hinich's (1978) Theorem 2, where he claims existence of a pure strategy equilibrium when bias terms are distributed with small enough variance. Hinich does prove that, for a fixed platform $x_{B}$ for candidate $B, A$ 's expected plurality maximizing response converges to $x_{B}$ as variance goes to zero, an intuitive result: as voters become more deterministic, candidate $A$ is better able to "capture" the voters to one side of $B$ and can then move toward $B$ to increase his/her plurality. Hinich continues the argument by fixing $x_{B}$ at the mean and proving that there is a certain open interval around $x_{B}$ within which the best response to $x_{B}$ is the mean itself (his Lemma). Letting the variance go to zero, Hinich reasons that the best response $x_{A}$ 's must eventually reach that open interval and must then be the mean itself. Thus, the mean would seem an equilibrium. But the open interval itself depends on the variance, and it collapses to the mean as the variance goes to zero. Hinich's proof breaks down because the best response $x_{A}$ does not reach the open interval.

In fact, the reverse of Hinich's claim is true: if the variance of the bias term is large enough, pure strategy equilibria will exist and they will be at the mean. From Theorem 2, we know that the key sufficient condition for existence of pure strategy equilibria is the concavity of the expected plurality shares as functions of policy platforms. Assume voter utility functions are quadratic and therefore concave (with second derivatives bounded below zero). When $G_{t}$ is the distribution of a normally distributed random variable, it will never be concave, but it will be arbitrarily close to concave as the variance of the distribution goes to infinity. Composed with the voters' utility functions, the candidates' expected plurality share functions will eventually be concave, as needed. Then Corollary 4 implies the equilibrium platforms are at the mean.

\section{$9 \quad$ The Utility Ratio Model}

Some of our results (namely, Theorems 8, 10-12 and Corollaries 1-4, 7, 8) are stated and proved for the special case of the Utility Difference Model, rather than the Utility Ratio Model. In this section, we will show that the two models are interchangeable, in a sense, and 
that corresponding results hold for the latter model. Given utility functions $u_{t}$ for voters and expected plurality share functions $\hat{P}_{t}^{C}$ in the Utility Ratio Model, define the following Utility Difference Model:

$$
\begin{aligned}
\dot{u}_{t}(x) & =\ln \left(u_{t}(x)\right) \\
\dot{P}_{t}^{C}(u) & =\hat{P}_{t}^{C}\left(e^{u}\right)
\end{aligned}
$$

for all $t$. The candidates' objective functions in the two models are clearly identical:

$$
\begin{aligned}
P_{t}^{C}\left(x_{A}, x_{B}\right) & =\hat{P}_{t}^{C}\left(u_{t}\left(x_{A}\right) / u_{t}\left(x_{B}\right)\right) \\
& =\dot{P}_{t}^{C}\left(\dot{u}_{t}\left(x_{A}\right)-\dot{u}_{t}\left(x_{B}\right)\right) .
\end{aligned}
$$

Furthermore, many properties of utility and expected plurality share functions in the Utility Ratio Model translate directly to properties in the associated Utility Difference Model. Continuity, differentiability, and monotonicity properties are preserved by the translation. Concavity of voter utility functions is clearly preserved, as well. The core is invariant under this transformation. And if $\left\{\hat{P}_{t}^{C, m}\right\}$ converges pointwise to $\hat{P}_{t}^{C}$, then $\left\{\dot{P}_{t}^{C, m}\right\}$ converges pointwise to $\dot{P}_{t}^{C}$.

As a consequence, Theorems 8 and 10-12 and Corollaries 7 and 8 carry over to the Utility Ratio Model directly: we need only replace "Utility Difference Model" with "Utility Ratio Model." To make this clear, consider the assumptions of the first part of Theorem 10 in the context of the Utility Ratio Model, i.e., $u_{t}(x, \lambda)$ jointly continuous, $\hat{P}_{t}^{C, m}$ weakly monotonic and converging pointwise to $\hat{P}_{t}^{C}$, pure strategy equilibria $\left(x_{A}^{m}, x_{B}^{m}\right)$ converging to $\left(x_{A}, x_{B}\right)$, etc. Now go to the associated Utility Difference Model: $\dot{u}_{t},\left\{\dot{P}_{t}^{C, m}\right\}, \dot{P}_{t}^{C}$. All of the assumptions of the first part of Theorem 10 hold, and the theorem yields $x_{A}$ and $x_{B}$ in the core defined using $\dot{u}_{t}$. Since the core is invariant under our transformation, we have $x_{A}$ and $x_{B}$ in the core of the original model. The same logic holds for the rest of Theorem 10.

In fact, Corollaries 1 and 2 carry over as well, in a way that highlights the connection between the Utility Difference and Utility Ratio Models. The next result follows directly from Corollary 1 by going to the associated Utility Difference Model. Let $\mathcal{U}_{t} / \mathcal{U}_{t}=\left\{u_{t}(x) / u_{t}(y) \mid x, y \in X\right\}$ denote the set of possible Utility Ratios for type $t$ voters.

Corollary $\mathbf{1}^{\prime}$ In the Utility Ratio Model, assume (i) $X$ is compact and convex, (ii) for each $t$ and $C, \hat{P}_{t}^{C}$ is continuous and weakly monotonic, (iii) for each $t, \hat{P}_{t}^{A}\left(e^{u}\right)$ is concave on $\mathcal{U}_{t} / \mathcal{U}_{t}$, (iv) for each $t, \hat{P}_{t}^{B}\left(e^{-u}\right)$ is concave on $\mathcal{U}_{t} / \mathcal{U}_{t}$, and (v) for each $t$, $u_{t}$ is continuous and $\ln \left(u_{t}\right)$ concave. Then there exists a pure strategy electoral equilibrium.

Assume, moreover, (vi) for each $t$ and $C, \hat{P}_{t}^{C}$ is strictly monotonic, and (vii) aggregate strict concavity holds with respect to logs of voter utility functions. Then there is exactly one electoral equilibrium, and it is in pure strategies.

The proof follows easily from Corollary 1: $\dot{u}_{t}$ is continuous and concave, and $\dot{P}_{t}^{C}(u)$ is weakly monotonic, continuous, and concave, so there is a pure strategy equilibrium of the associated Utility Difference Model. Since candidates' payoffs are invariant under the transformation, we have an equilibrium of the original model. The second part also follows directly from Corollary 1 . The next result follows directly from Corollary 2 . 
Corollary $2^{\prime}$ In the Utility Ratio Model, assume (i) $X$ is compact and convex, (ii) for each $t$ and $C, \hat{P}_{t}^{C}(u)$ is continuous and weakly monotonic on $\mathcal{U}_{t} / \mathcal{U}_{t}$, (iii) for each $t, \hat{P}_{t}^{A}(u)$ is concave on $\mathcal{U}_{t} / \mathcal{U}_{t}$, (iv) for each $t, \hat{P}_{t}^{B}\left(\frac{1}{u}\right)$ is concave on $\mathcal{U}_{t} / \mathcal{U}_{t}$, and (v) for each $t, u_{t}$ is continuous and concave. Then there exists a pure strategy electoral equilibrium.

Assume, moreover, (vi) for each $t$ and $C, \hat{P}_{t}^{C}$ is strictly monotonic, and (vii) aggregate strict concavity holds. There is exactly one electoral equilibrium, and it is in pure strategies.

For an example of expected plurality share functions in the Utility Ratio Model satisfying (ii)-(iv) and (vi) from Corollary $2^{\prime}$, consider

$$
\hat{P}_{t}^{A}(u)=\frac{u-1}{u+1} \quad \text { and } \quad \hat{P}_{t}^{B}(u)=\frac{1-u}{u+1} .
$$

Note that these functional forms give us

$$
\begin{aligned}
P_{t}^{A}\left(x_{A}, x_{B}\right) & =\frac{u_{t}\left(x_{A}\right)}{u_{t}\left(x_{A}\right)+u_{t}\left(x_{B}\right)}-\frac{u_{t}\left(x_{B}\right)}{u_{t}\left(x_{A}\right)+u_{t}\left(x_{B}\right)} \\
P_{t}^{B}\left(x_{A}, x_{B}\right) & =\frac{u_{t}\left(x_{B}\right)}{u_{t}\left(x_{A}\right)+u_{t}\left(x_{B}\right)}-\frac{u_{t}\left(x_{A}\right)}{u_{t}\left(x_{A}\right)+u_{t}\left(x_{B}\right)},
\end{aligned}
$$

which is just the Binary Luce Model used by Coughlin and Nitzan (1981) and Coughlin (1992). Thus, we have derived their existence and uniqueness result from results for the Utility Difference Model, under minimal assumptions - in particular, their assumption of the specific Binary Luce functional form is unneeded.

We can also derive their characterization in terms of Nash, rather than utilitarian, social welfare optima. Again, their result follows quite generally, relying only on the structure of the Utility Ratio Model - the Binary Luce functional form is unneeded.

Corollary $3^{\prime}$ In the Utility Ratio Model, assume (i) $X$ is convex, (ii) for each $t$ and $C$, $\hat{P}_{t}^{C}(u)$ is strictly monotonic and differentiable with derivatives bounded in $(t, u)$ and with $D_{u=1} \hat{P}_{t}^{C}(u)$ independent of $t$, and (iii) for each $t, \ln \left(u_{t}\right)$ is concave. If $\left(x^{*}, x^{*}\right)$ is a pure strategy equilibrium of the electoral game, then $x^{*}$ maximizes

$$
\int_{T} \ln \left(u_{t}(x)\right) \mu(d t)
$$

Assume, moreover, (iv) aggregate strict concavity holds with respect to logs of voter utility functions. Then $\left(x^{*}, x^{*}\right)$ is the unique interior pure strategy electoral equilibrium exhibiting policy coincidence.

The result for the Utility Ratio Model follows by transforming the model into its associated Utility Difference Model. Doing so, Corollary 3 implies that $x^{*}$ maximizes

$$
\int_{T} \dot{u}_{t}(x) \mu(d t)=\int_{T} \ln \left(u_{t}(x)\right) \mu(d t),
$$

and it implies the remainder of the theorem. We started with the Utility Difference Model because we appreciated the interpretation of the Additive Bias Model, but from a technical 
perspective we could equally well have begun with the Utility Ratio Model: any result for one model translates into a result for the other. More importantly, the apparent conflict between theories that predict utilitarian optima and those that predict Nash optima is resolved as a matter of modelling preference.

\section{Summary}

We have presented numerous results predicated on numerous assumptions, those results often stated in a general form and then specialized to the case of the Utility Difference Model (or the Utility Ratio Model), which has the clear interpretation in terms of a random Additive Bias term for each voter. We end with a quick summary of our results for the Utility Difference Model, under the following minimal background assumptions. When they hold, our results for the Utility Difference Model are quite sharp.

- $X$ is compact and convex.

- For each $t$ and $C, \hat{P}_{t}^{C}$ is strictly monotonic and differentiable with derivatives bounded in $(t, u)$ and with $D_{u=0} \hat{P}_{t}^{C}(u)$ independent of $t$.

- For each $t, u_{t}$ is continuous and concave.

- Aggregate strict concavity holds.

The above conditions on expected plurality share functions hold, for example, in the Additive Bias Model whenever voter biases are identically distributed according to a continuous, strictly increasing distribution function.

Under these assumptions, Theorem 1 yields the existence of a mixed strategy electoral equilibrium. If a pure strategy electoral equilibrium exists, then, by Theorem 8 , it exhibits policy coincidence. By Corollary 3, the policy platform adopted by the candidates in any such equilibrium maximizes the utilitarian social welfare function.

When do pure strategy equilibria exist? If nothing more than concavity of the voters' utility functions is known, we must assume the expected plurality share functions of the candidates are affine linear, as when the Additive Bias term for each voter type is uniformly distributed. In that case, Corollary 1 gives us existence and uniqueness of a pure strategy equilibrium. Moreover, as discussed after Theorem 4, that equilibrium platform is actually a dominant strategy for the candidates, in the sense that it is a best response to every platform one's opponent might adopt. Otherwise, if it is known that voter utilities possess stronger concavity properties, then weaker conditions may be imposed on expected plurality share functions, as illustrated in Corollary 2.

Pure strategy electoral equilibria will not exist, by Theorem 12, if the core is non-empty, it does not include the utilitarian optimum, and the expected plurality share functions are close enough to the Deterministic Model. In the one-dimensional model with an odd number of voters and quadratic utilities, this simplifies to the following: if the mean ideal point is distinct from the median, then in models close enough to deterministic, pure strategy 
equilibria will not exist. From Theorem 12, however, mixed strategy electoral equilibria will exist, and the equilibrium mixed strategies of the candidates must put arbitrarily high probability near the median.

\section{References}

[1] C. Aliprantis and K. Border (1994) Infinite Dimensional Analysis: A Hitchhiker's Guide, New York: Springer-Verlag.

[2] C. Aliprantis and O. Burkinshaw (1990) Principles of Real Analysis, New York: Academic Press.

[3] R. Ball (1999) "Discontinuity and Non-existence of Equilibrium in the Probabilistic Spatial Voting Model," Social Choice and Welfare, 16:533-556.

[4] J. Banks and J. Duggan (1998) "Existence of Nash Equilibria on Convex Sets," mimeo.

[5] J. Banks and J. Duggan (2002) "A Multi-dimensional Model of Repeated Elections," mimeo.

[6] J. Banks, J. Duggan, and M. Le Breton (2003) "Social Choice and Electoral Competition in the General Spatial Model," mimeo.

[7] T. Besley and S. Coate (1997) "An Econoimc Model of Representative Democracy," Quarterly Journal of Economics, 112:85-114.

[8] D. Black (1958) The Theory of Committees and Elections, Cambridge: Cambridge University Press.

[9] R. Calvert (1985) "Robustness of the Multidimensional Voting Model: Candidate Motivations, Uncertainty, and Convergence," American Journal of Political Science, 29:6995.

[10] P. Coughlin (1992) Probabilistic Voting Theory, Cambridge: Cambridge University Press.

[11] P. Coughlin and S. Nitzan (1981) "Electoral Ourcomes with Probabilistic Voting and Nash Social Welfare Maxima," Journal of Public Economics, 15:113-121.

[12] A. Downs (1957) An Economic Theory of Democracy, New York: Harper and Row.

[13] J. Duggan (2000) "Equilibrium Equivalence under Expected Plurality and Probability of Winning Maximization," mimeo.

[14] M. Hinich (1977) "Equilibrium in Spatial Voting: The Median Voter Result is an Artifact," Journal of Economic Theory 16:208-219.

[15] M. Hinich (1978) "The Mean Versus the Median in Spatial Voting Games," in P. Ordeshook, ed., Game Theory and Political Science, New York: NYU Press. 
[16] M. Hinich, J. Ledyard, and P. Ordeshook (1972) "Nonvoting and the Existence of Equilibrium Under Majority Rule," Journal of Economic Theory, 4:144-153.

[17] M. Hinich, J. Ledyard, and P. Ordeshook (1973) "A Theory of Electoral Equilibrium: A Spatial Analysis Based on the Theory of Games," Journal of Politics, 35:154-193.

[18] H. Hotelling (1929) "Stability in Competition," Economic Journal, 39:41-57.

[19] G. Kramer (1978) "Robustness of the Median Voter Result," Journal of Economic Theory, 19:565-567.

[20] J. Ledyard (1984) "The Pure Theory of Large Two-candidate Elections," Public Choice, 44:7-41.

[21] A. Lindbeck and J. Weibull (1987) "Balanced-budget Redistribution as the Outcome of Political Competition," Public Choice, 52:273-297.

[22] A. Lindbeck and J. Weibull (1993) "A Model of Political Equilibrium in a Representative Democracy," Journal of Public Economics, 51:195-209.

[23] R. McKelvey and J. Patty (2003) "A Theory of Voting in Large Elections," mimeo.

[24] M. Osborne and A. Slivinski (1996) "A Model of Political Competition with CitizenCandidates," Quarterly Journal of Economics, 111:65-96.

[25] J. Patty (2002) "Equivalence of Objectives in Two Candidate Elections," Public Choice, 112:151-166.

[26] J. Patty (2003) "Local Equilibrium Equivalence in Probabilistic Voting Models," mimeo.

[27] C. Plott (1967) "A Notion of Equilibrium and Its Possibility Under Majority Rule," American Economic Review, 57:787-806.

[28] J. Rosen (1965) "Existence and Uniqueness of Equilibrium Points for Concave $n$-person Games," Econometrica, 33:520-534. 